\title{
Research Paper \\ Validity of the Integrated Motivational-Volitional Model of Suicidal Behavior in Students: Structural Model
}

\author{
*Ahmadreza Kiani Chelmardi ${ }^{1}$ (10, Sajjad Rashid² ${ }^{2}$, Shoukofeh Ramezani ${ }^{3}$ (1)
}

1. PhD. in Family Counseling, Assistant Professor, Department of Counseling, Faculty of Education and Psychology, University of Mohaghegh Ardabili, Ardabil, Iran 2. PhD. Student of Medicine, Department of International Education, China Medical University, Shenyang, China.

3. MA. in Rehabilitation Counseling, Department of Counseling, Faculty of Education and Psychology, University of Mohaghegh Ardabili, Ardabil, Iran

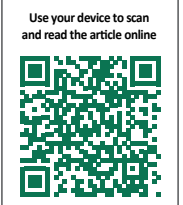

Citration Kiani Chelmardi AR, Rashid S, Ramezani Sh. Validity of the Integrated Motivational-Volitional Model of Suicidal Behavior in Students: Structural Model. Iranian Journal of Psychiatry and Clinical Psychology. 2019; 25(2):194-209. http://dx.doi. org/10.32598/ijpcp.25.2.194

http://dx.doi.org/10.32598/ijpcp.25.2.194

Received: 11 Aug 2018

Accepted: 27 Feb 2019

Available Online: $01 \mathrm{Jul} 2019$

Key words:

Suicide, Integrated Motivational-Volitional Model, Defeat, Entrapment, Impulsivity, Interpersonal Needs Questionnaire

\section{AB STRACT}

Objectives The current study aimed at validating the Integrated Motivational-Volitional Model of suicidal behavior in Iranian students.

Methods The population of the current descriptive correlational and structural equation model included all students of Ardabil city among which 520 people of Mohaghegh Ardabili University students were selected as the sample. Suicide Attempt Scale, The Suicide Behaviors Questionnaire-Revised (SBQ-R), Implementation Scale, The Short Defeat and Entrapment Scale, Interpersonal Needs Questionnaire, Impulsivity Scale, Exposure to suicidal behavior Scale, Self-Perceived Acquired Capability for Suicide Scale, and Non-suicidal self-injury Scale were used.

Results The study findings showed that the modal had a good fitness in students' community and can explain suicide properly. Defeat and Entrapment play the most roles in forming suicide ideas and interpersonal needs; loneliness has a little effect on forming suicide ideas. Implementation, acquired capability for suicide and suicide ideas are the most important mediators that can explain suicide attempt in this model.

Conclusion Based on the current study findings it can be concluded that Motivational Volitional Model can explain suicide.

\section{Extended Abstract}

\section{Introduction}

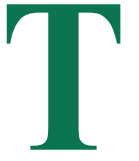

he current study aimed at validating the Integrated Motivational- $\mathrm{V}$ olitional model of suicidal behavior in Iranian Students. Suicide is a leading cause of death worldwide, killing more than 800,000 people each year. with some researchers estimating that approximately 25 attempts occur for every suicide death. Recent researches suggest that the rate of suicide in Iran increased to 9.9 in the past two decades. Also, 20-25 non-fatal suicide attempts occurred for every fatal one. Therefore, estimate would show that more than 198000 people committed suicide in 2016 in Iran and developing in-depth knowledge for suicide is an imperative issue for Iran. Especially for college students who are more vulnerable to suicide.

A much larger number of people make suicide attempts,

* Corresponding Author:

Ahmadreza Kiani Chelmardi, PhD.

Address: Department of Counseling, Faculty of Education and Psychology, University of Mohaghegh Ardabili, Ardabil, Iran. Tel: +98 (911) 2521205

E-mail: a.kiani@uma.ac.ir 
In the past two decades, numerous models of suicidal behavior were formed and led to important developments in understanding the etiology and course of suicidal behavior. As a consequence, there is growing recognition that it is needed to move beyond the classic psychiatric diagnostic categories to further understand the causes of suicidal behavior.

The Integrated Motivational-Volitional (IMV) Model of Suicidal Behavior aimed at discriminating between suicide ideators and suicide attempters. The IMV proposes that suicidal behavior results from a complex interplay of factors, the proximal predictor of which is one's intention to engage in suicidal behavior (behavioral intention). Behavioral intention, subsequently, is determined by feelings of entrapment where suicidality is observed as the salient solution to life circumstances and entrapment is triggered by defeat/humiliation appraisals. The transitions from the defeat/humiliation to entrapment, from entrapment to suicidal ideation/intent, and from ideation/intent to suicidal behavior are determined by state-specific moderators (i.e. factors which facilitate/obstruct movement between states), entitled threat to self, motivational, and volitional moderators, respectively. In addition, background factors (e.g. deprivation, vulnerabilities) and life events (e.g. relationship crises), which collectively comprise the pre-motivational phase (i.e. before the commencement of ideation formation), provide the broader biosocial context for suicide.

Within the IMV model, it is hypothesized that sensitivity to signals of defeat and humiliation are determined by background factors (pre-motivational phase). As an example, this sensitivity to signals of defeat may be increased by what we believe others expect us. In suicidal individuals, such expectations are often excessive and unrealistic, with the suicidal individual believing that they will be considered a failure if they do not achieve certain standards. Although entrapment can be triggered by defeat/humiliation, it is not inevitable that the experience of defeat/humiliation lead to entrapment: Threat to Self-Moderators (TSM) have the potential to increase the likelihood that the former leads to feelings of entrapment. TSMs are defined as any variable that attenuates or strengthens the relationships between threat to self-appraisals, that is, defeat/humiliation, and entrapment. Components within the TSM are activated in response to a defeating or humiliating appraisal (usually following the experience of negative life stress or a change in severity of existing chronic stress).

Social problem-solving is a likely candidate as a moderator of the defeat-entrapment relationship, since it is activated in an attempt to resolve the defeating/humiliating situation. Over-general autobiographical memory biases are also included here since they are known to be associated with impaired social problem-solving. The evidence implicating social problem solving and autobiographical memory in the suicidal process is well established. Over-general memories are incomplete, lack specific detail, and often refer to activities/objects without specifying a time period or covering a time period greater than a day. Since people heavily rely on memories to solve interpersonal problems, it is easy to see how this over-general memory bias impairs problem-solving and is more generally implicated in emotional distress.

The IMV model suggests that the presence/absence of Motivational Moderators (MM) increases/decreases the likelihood that entrapment is translated into suicidal ideation and intent. A motivational moderator is broadly defined as any factor (moderator) that changes the likelihood that entrapment leads to suicidal ideation and intent. Absence of positive future thinking, lack of goal re-engagement, and low levels of social support are typical of motivational moderators-as they increase the likelihood that suicidal ideation develops following entrapment. They would include what Williams (2001) described as rescue factors in his arrested flight model. In the following section, some of the evidence for positive future thinking and goal re-engagement are considered.

\section{Method}

Structural Equation Modeling (SEM) was conducted to examine direct and indirect pathways of the study's model; 520 college students were recruited from the University of Mohaghegh Ardabili. Their age ranged from 17 to 48 years $(\mathrm{M}=22.4 ; \mathrm{SD}=3.8)$. Nearly $60 \%$ of participants were from Ardebil city, Iran and approximately $40 \%$ were from other provinces . Only $17 \%$ said that they had any income. Most of the students were bachelor students (87\%), not married (79\%), and female (70\%).

Measures of the current study included Suicide A ttempt, single item adapted from Nock et al. used to assess suicide attempt history. The study used "how many times have you" before "made an actual attempt to kill yourself in which you had at least some intent to die?". The Suicide Behaviors Questionnaire-Revised (SBQ-R) included four items to assess lifetime suicide ideation and/or attempt, suicidal ideation in the past year, the threat of suicide, and the likelihood of committing suicide in future. Implementation, based on the definition by Gollwitzer, 1999, a one-item question was developed: "Have you ever had a plan to kill yourself at a specific time (e.g. Monday or even specific time), a specific place (e.g. home, street), by a specific method (egdrug overdose)?". The Short Defeat and Entrapment Scale is an eight-item scale assessing defeat 
and entrapment as a unified concept. Interpersonal Needs Questionnaire (INQ) contains subscales for Perceived Burdensomeness (7 items; e.g. "These days I think my death would be a relief to the people in my life) and thwarted belongingness ( 8 items; e.g. "These days, I often feel like an outsider in social gatherings"). Impulsivity, two items based on research by O'Connor et al. were selected from the Plutchik Impulsivity Scale to assess this construct. Exposure to suicidal behavior included two questions about selfharm by close friends and family, Self-Perceived Acquired Capability for Suicide 2016) that include 1 item question "I could kill myself if I wanted to", and Non-suicidal selfinjury included three items adapted from the non-suicidal self-injury assessment tool.

\section{Discussion}

Findings showed that this modal had a good fitness in Iranian community and could predict suicide properly. Defeat and Entrapment $(\mathrm{r}=0.36)$ play the most important roles in forming suicide ideas and interpersonal needs $(\mathrm{r}=0.24)$, loneliness has a little effect on forming suicide ideas. Implementation $(\mathrm{r}=0.16)$, acquired capability for suicide $(\mathrm{r}=0.14)$, and suicide ideas $(\mathrm{r}=0.24)$ are the most important mediators that can explain suicide attempt in this model.

Based on the study findings, it can be concluded that Motivational Volitional Model can predict suicide. Defeat and Entrapment play most of the roles in forming suicide ideas. In line with previous research, significant relationships were observed between defeat and entrapment with suicidal ideation, and exposure to self-injurious behavior in others and fearlessness about death and suicide attempts. The current article described the theoretical origins of the IMV model, the key premises underpinning the model, empirical tests of the model and future research directions. Finally, suitable intervention, especially cognitive behavioral strategies can be used to cut the link between pre-motivational and motivational phase with volitional phase.

\section{Ethical Considerations}

\section{Compliance with ethical guidelines}

The participants signed informed consent forms and had the right to leave the study at any time.

\section{Funding}

This research did not receive any specific grant from funding agencies in the public, commercial, or not-for -profit sectors. The paper was extracted from the ideas of the researchers about the students' suicide in the University of Mohaghegh Ardabili.

\section{Authors contributions}

Conceptualization: Ahmadreza Kiani Chelmardi, Sajjad Rashid; Investigation: Ahmadreza Kiani Chelmardi; Methodology: Sajjad Rashid; Writing and supervision: Ahmadreza Kiani Chelmardi, Resources: Shokoufe Ramezani.

\section{Conflicts of interest}

The authors declared no conflict of interests. 


\title{
اعتباريابى مدل يكيارجه انتَيزشى ارادى خودكشى در بين دانشجويان: مدل ساختارى
}

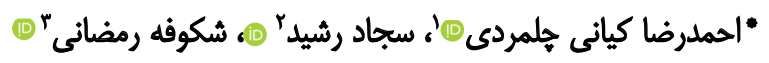

\author{
1- د دكثراي مشاوره خانواده، استاديار، كروه مشاوره، دانشكده علوم تربيتى و روانشناسى، داتشكاه محقق اردبيلى، اردبيل، ايران.

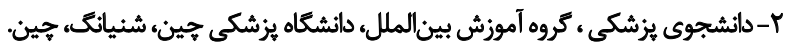

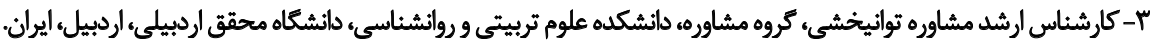

\begin{abstract}
حكيد

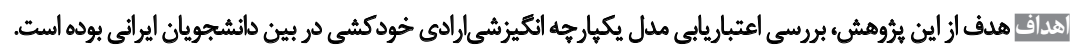

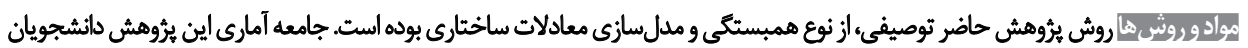

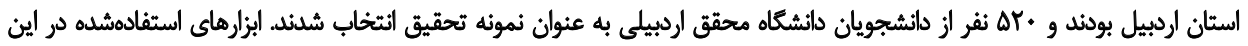

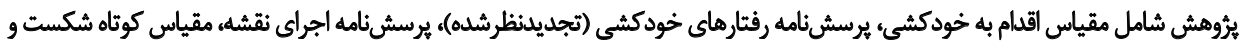

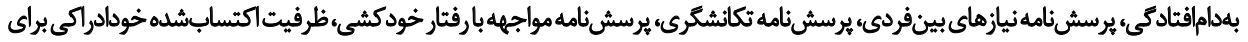

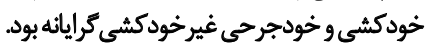

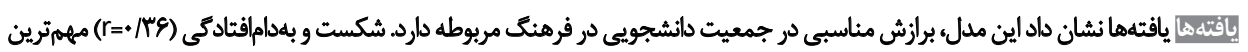

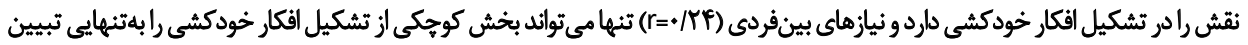

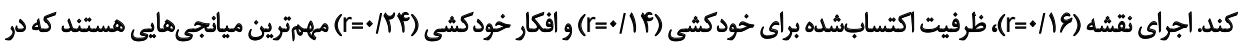

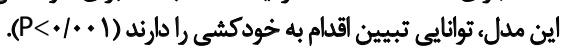

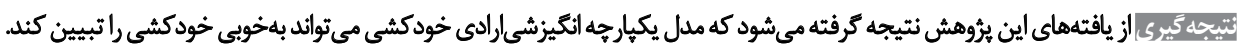

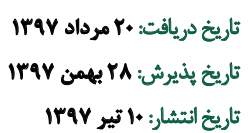

كليدوازوها: خودكشي، مدل يكيارجه انتيزشىارادى، شكست،

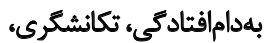

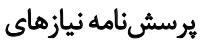
بينفردى

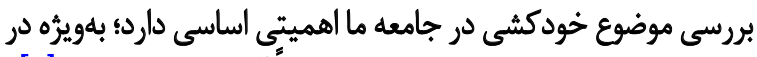

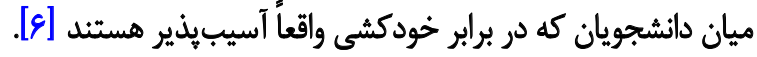

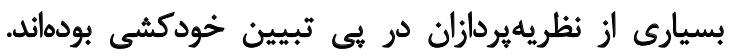

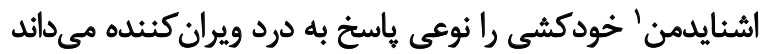

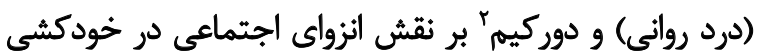

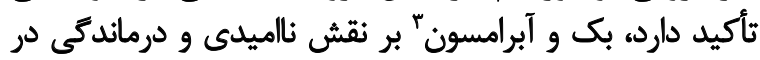

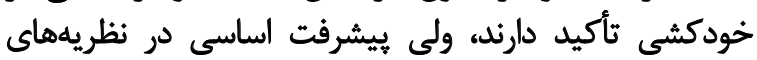

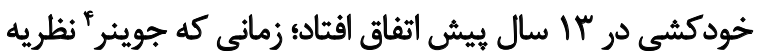

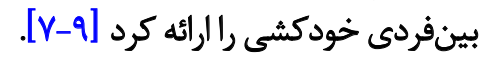
اين نظريه به صورت جامع و كستردهاى تعامل دوسويه بين

1. Shneidman

2. Durkheim

3. Beck and Abramson

4. Joiner dales هر ساله تقريباً يك ميليون نفر در سرثاسر جهان بر اثر

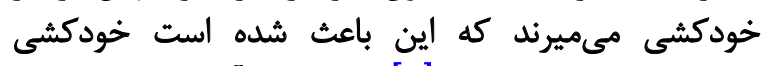

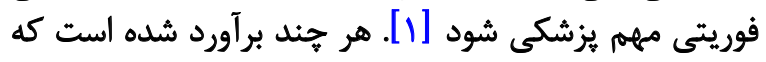

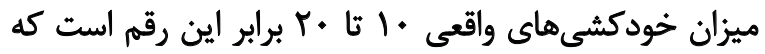

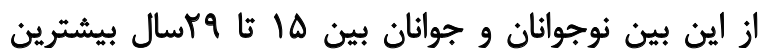

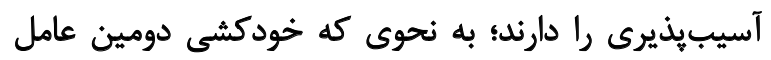

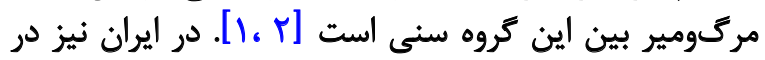

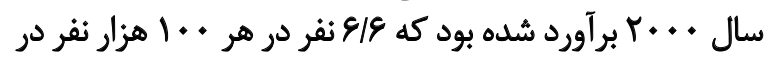

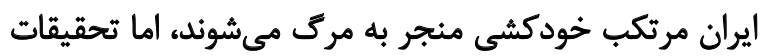

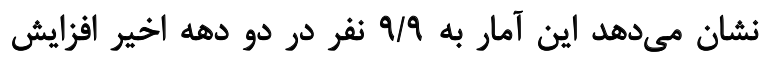

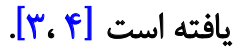
همجئين، در برابر يك اقدام به خودكشى موفق و منجر به مرك،

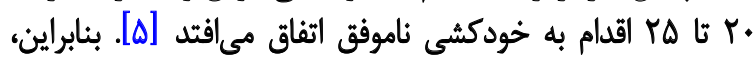

$$
\begin{aligned}
& \text { * : نويسئده مسينول: }
\end{aligned}
$$

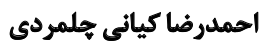

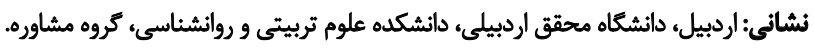

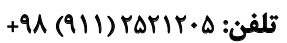

$$
\begin{aligned}
& \text { a.kiani@uma.ac.ir بلكت الكترونيكي }
\end{aligned}
$$




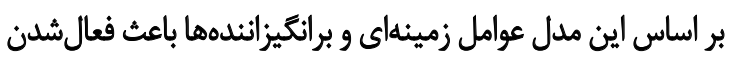

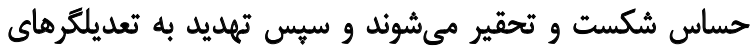

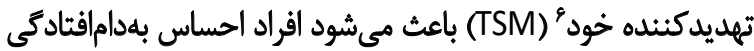

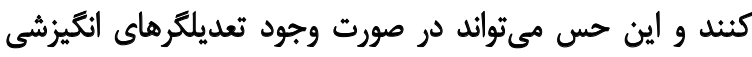

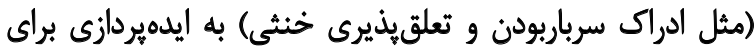

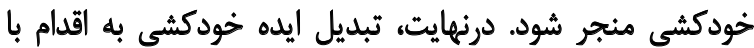

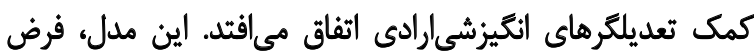

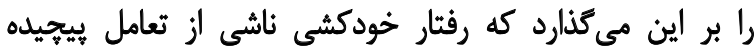

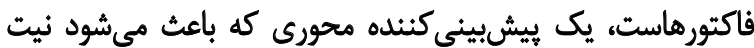

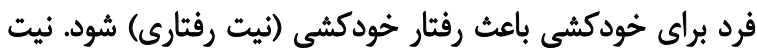

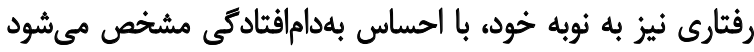

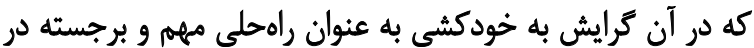

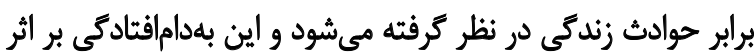

$$
\text { شكست و تحقير فعال مى دشود. }
$$

6. Threat to Self-Moderators (TSMs)
سيستمهاى يويايى درونفردى و عوامل خطر بينفردى را مد مد بد

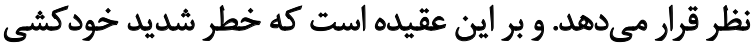

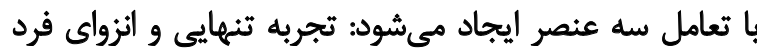

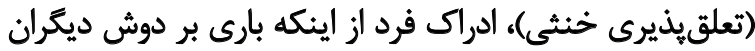

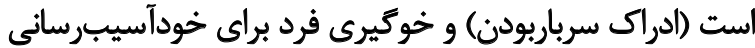

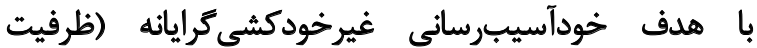

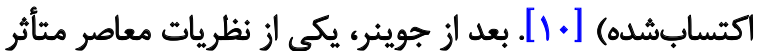

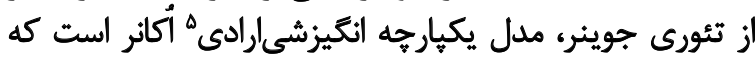

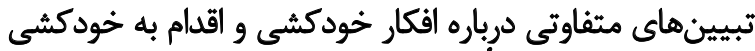

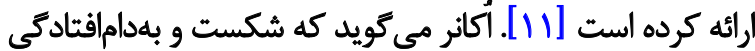

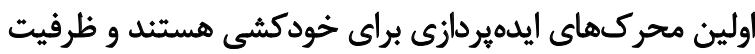

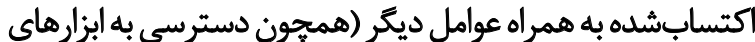

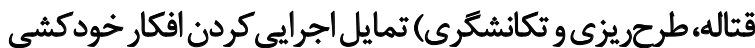

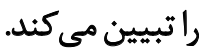

5. Integrated Motivational-Volitional (IMV) Model

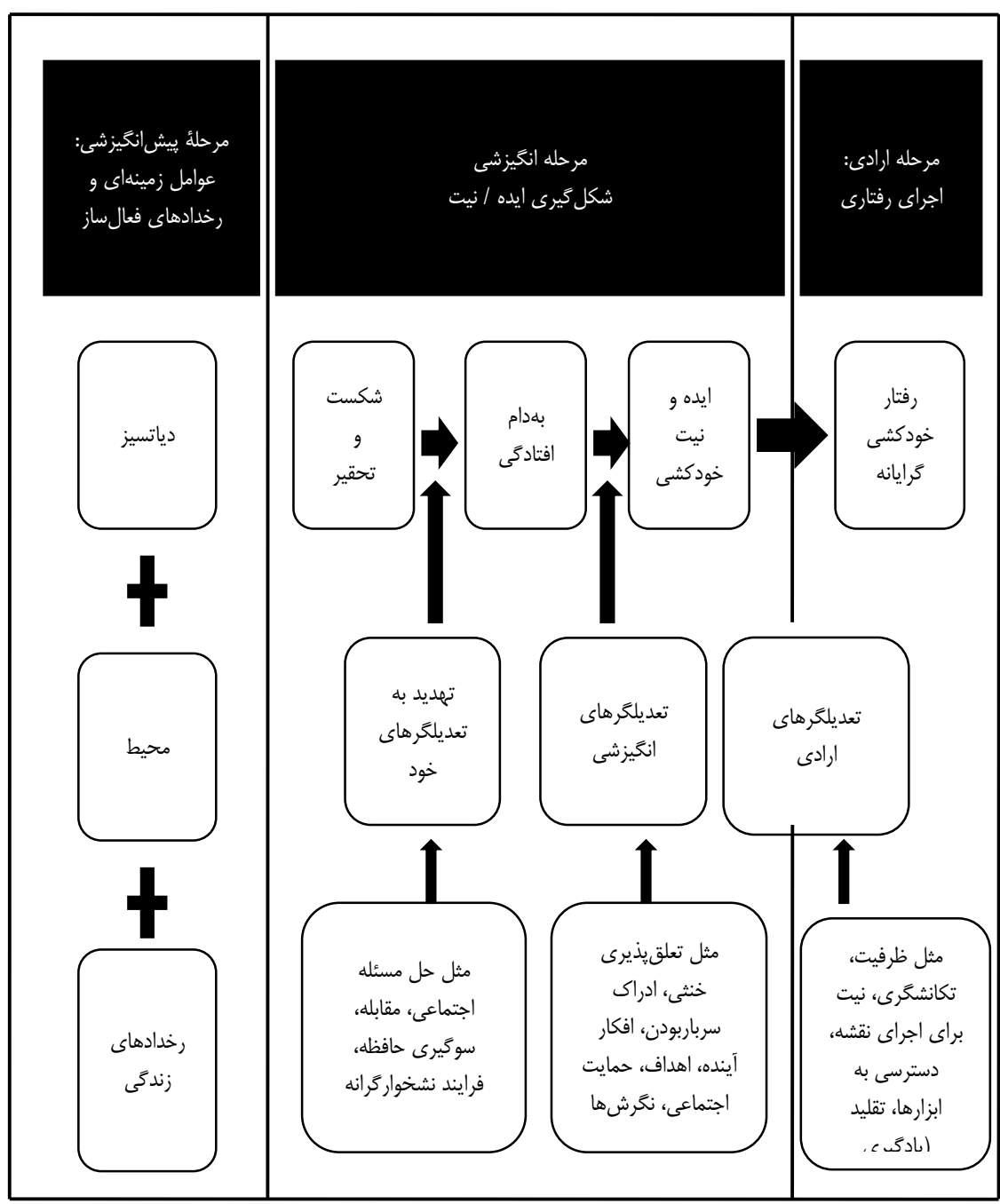




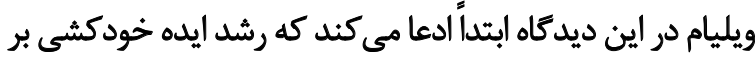

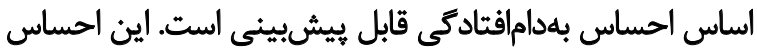

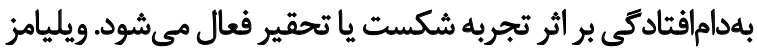

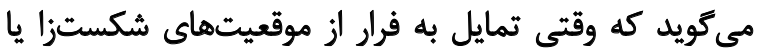

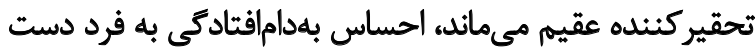

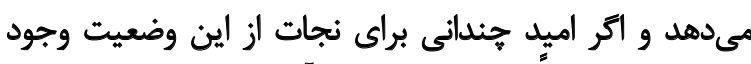

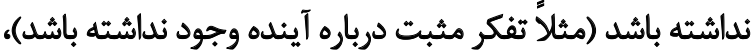

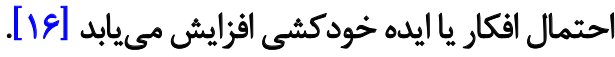

$$
\text { أشكست و تحقير در مدال IMV }
$$

فرض بر اين است كه حساسيت به علائم شكست و تحقير تحت

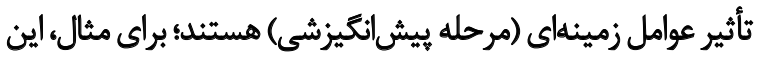
حساسيت به علاثم شكست ممكن است بر اثر ادراك ما ما از انتظاراتي

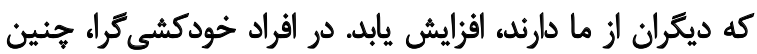

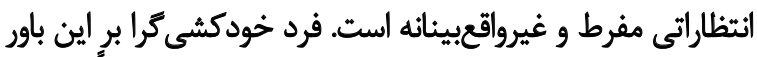

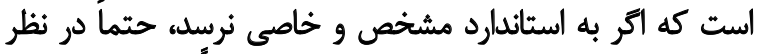

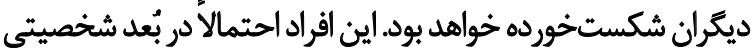

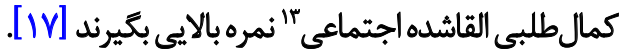

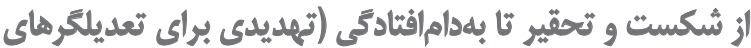
تهبديدكثندهود)

كرجه بهدام|فتادكى مي تواند بر اثر شكست/تحقير فعال شود، ولى

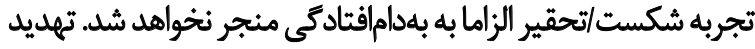

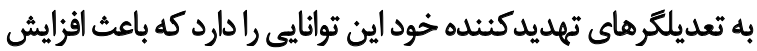

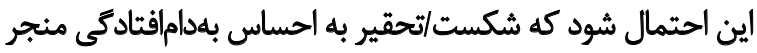

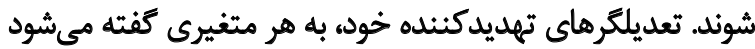

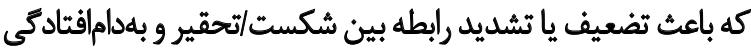

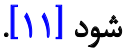

\section{از بلدامافتادئى ثاليده/ثيت خودكشى (تعديلكرهاى انتَيزشى)}

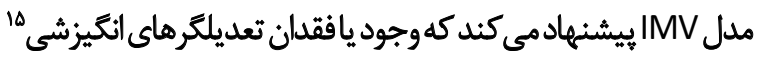

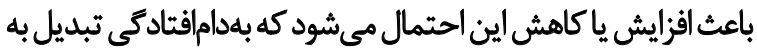

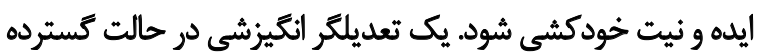

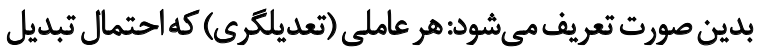

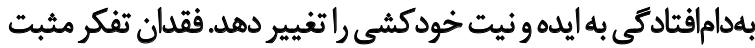

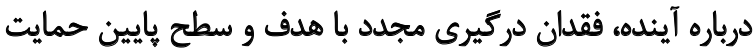

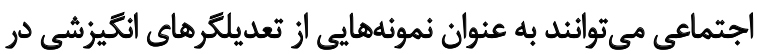

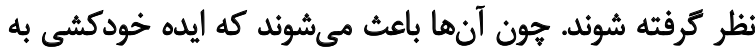

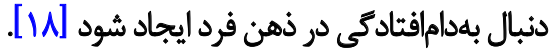

13. Socially prescribed perfectionism

14. Self moderators

15. Motivational Moderators (MM)
اساساً، عبور از مرحله شكست و تحقير و ورود به هرحله

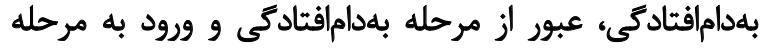

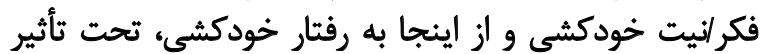

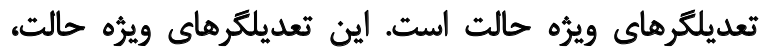

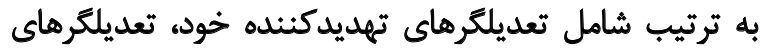

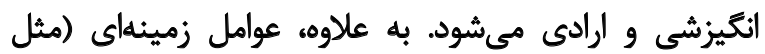

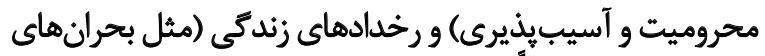

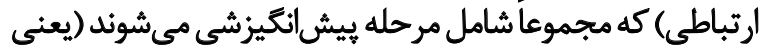

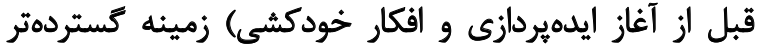

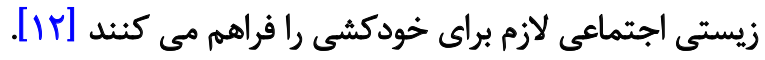

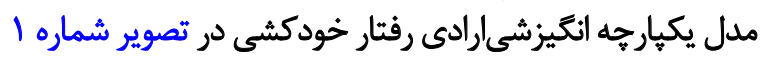
آورده شده است [11]].

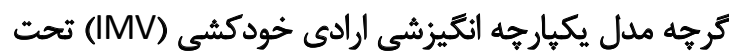

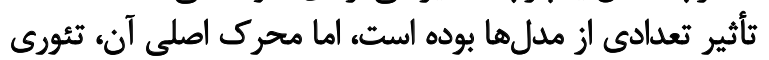

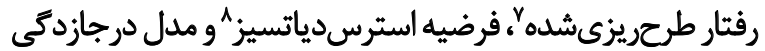

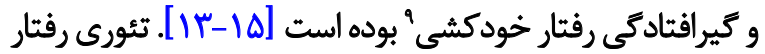

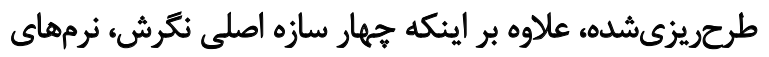

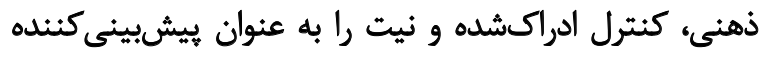

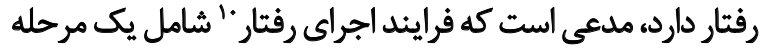

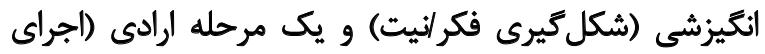
رفتارى) است. مرحله انكيزشي و ارادى اين مدل، در خلأ زيستى "اجتماعي

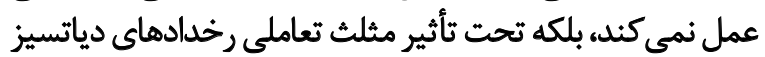

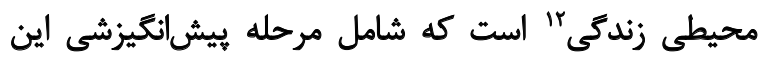

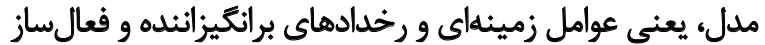

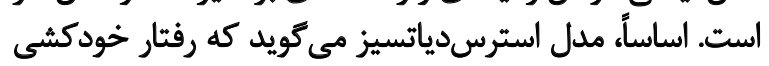

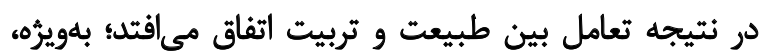

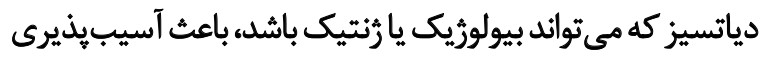

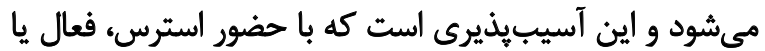

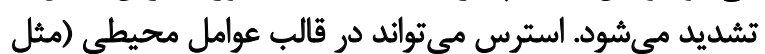

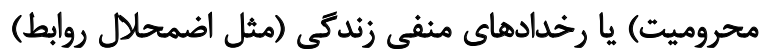

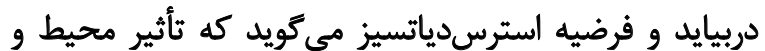

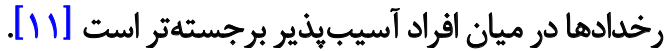

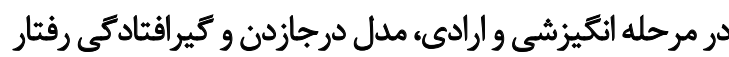

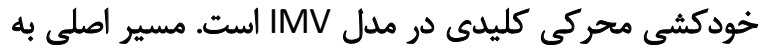

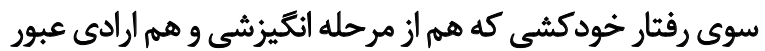

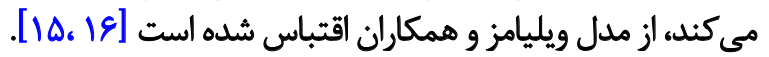

7. Theory of Planned Behaviour

8. Diathesis-Stress Hypothesis

9. Arrested Flight Model of Suicidal Behaviour

10. Behavioural enaction

11. Biosocial vacuum

12. Interactive diathesis-environment-life events triad 


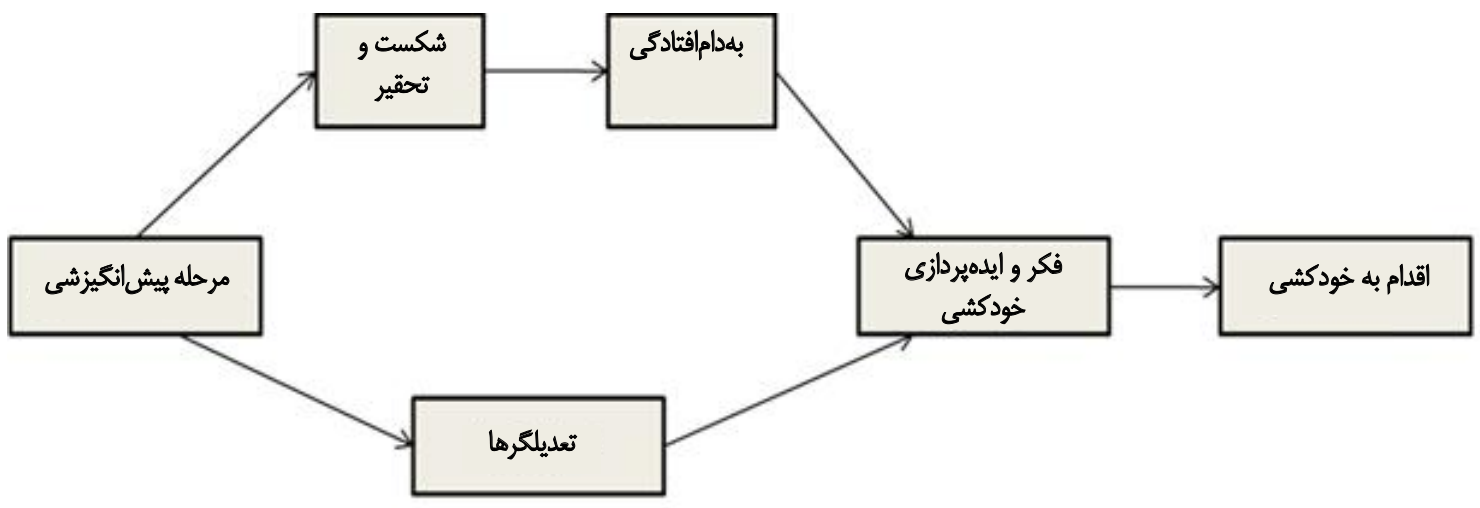

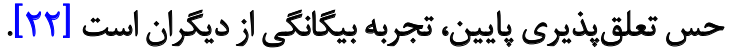

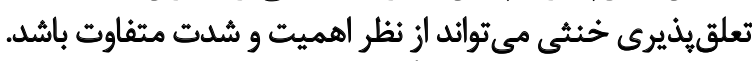

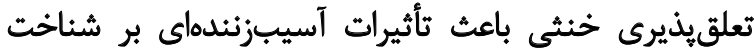

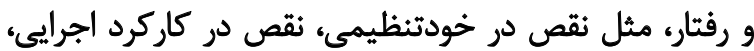

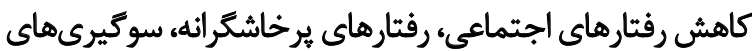

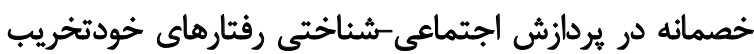

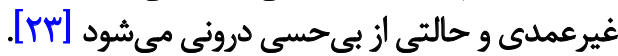

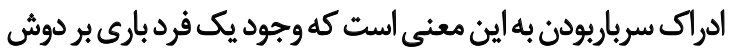

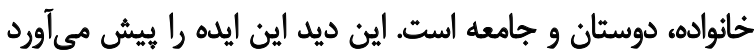

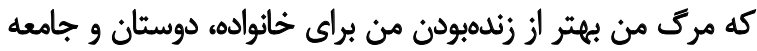

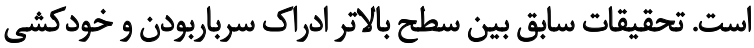

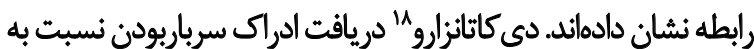

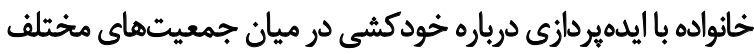

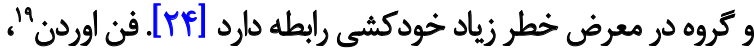

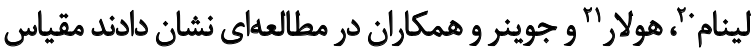

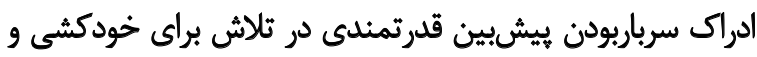
ايدهيردازى خودكشى است [Tهارئ.

با توجه به اهميت زياد خودكشى به عنوان يك آسيب اجتماعى

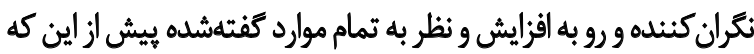

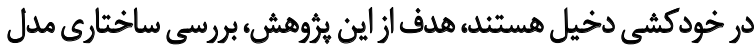

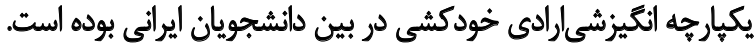
مدل اوليه مفروض نيز در تصوير شماره ب آمده است دئ.

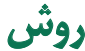

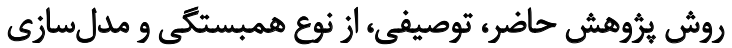

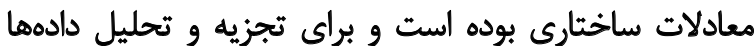

18. De Catanzaro

19. Van Orden

20. Lynam

21. Hollar

\section{از ايده/ثيت خُودكشى تا رفتار خُودكشى (مدل ارادى)}

اين بخش نحوه تبديل نيت خودكشى به رفتار خودكشى را رال

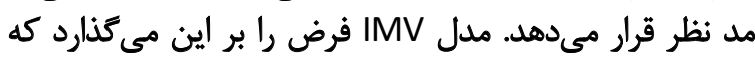

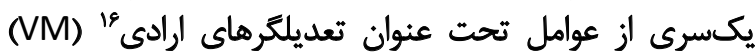

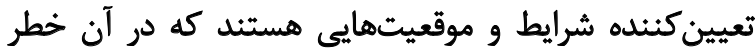

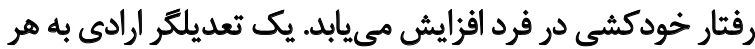

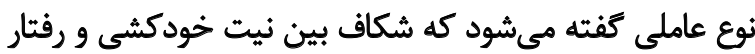

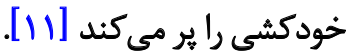

درنهايت، مفهوم ظرفيت اكتسابشده جوينر يك تعديلكًر

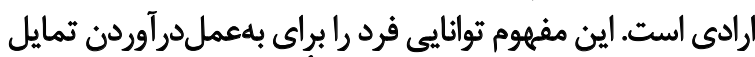

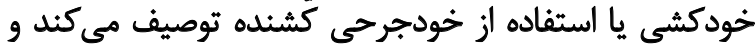

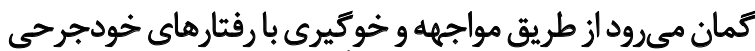

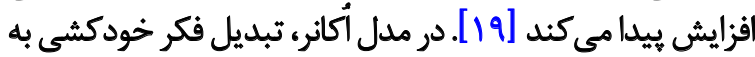

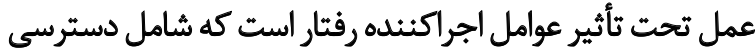

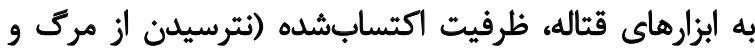

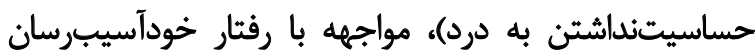

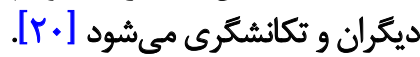
دراينجا،نظريهجوينرمطرحمى مود.نظريابينفردىروانشناختى

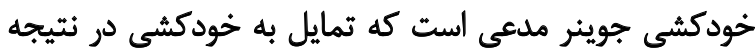

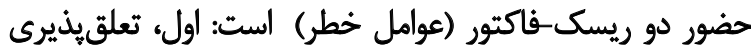

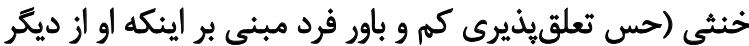

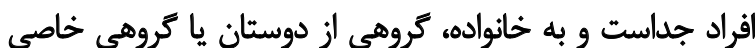

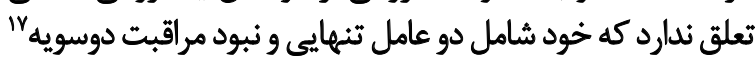

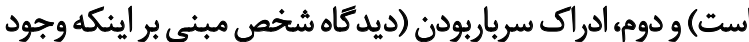

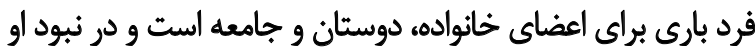

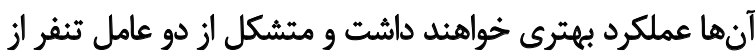

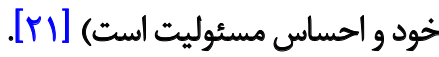

16. Volitional Moderators

17. Absence of reciprocal care 
غيرخودكشى كرا V است. مطالعه اصلى نشان از روايى همعراى

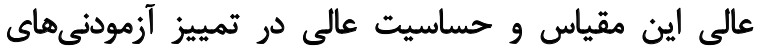

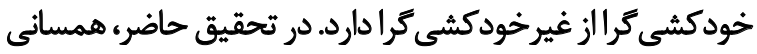

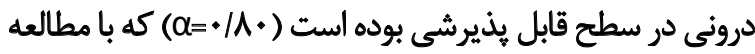

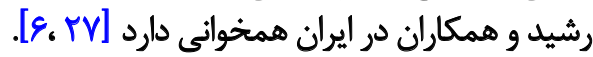

\section{يرسش نامه اجراي نقشه"}

بر مبناى تعريف تولويتزر بَّ، ما يك سؤال طرح كرديم: آيا

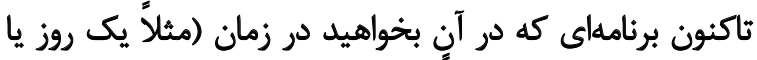

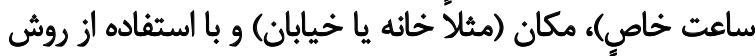

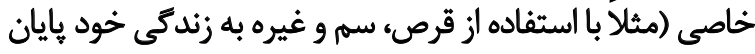

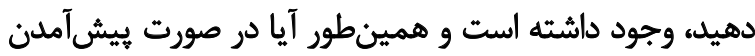

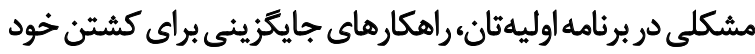

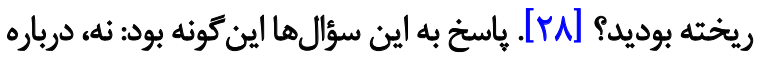

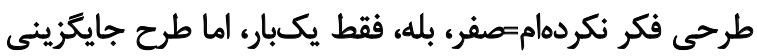

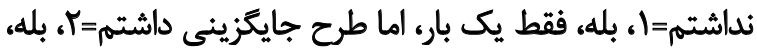

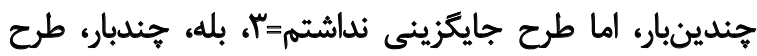

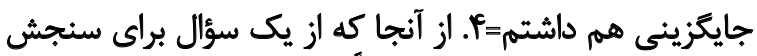

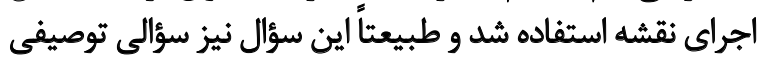

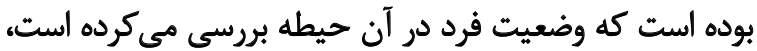

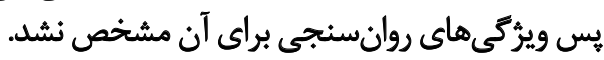

\section{مقياس كوثاه شكست و بهدامافتادتى}

اين يك مقياس هشتآيتمى است كه به ارزيابي شكست و

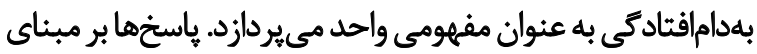

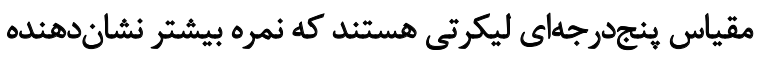

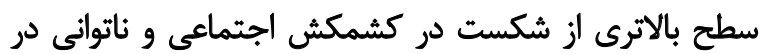

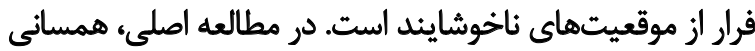

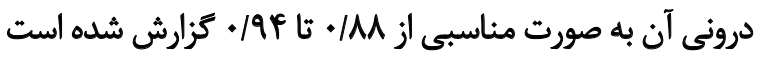

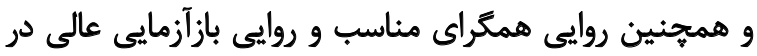

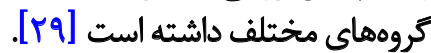

برسش نامهنيازهاى بينفردى

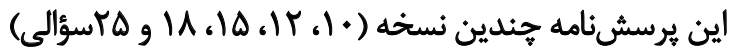

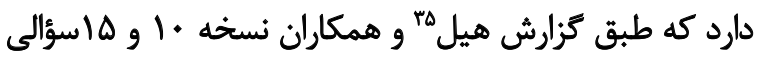

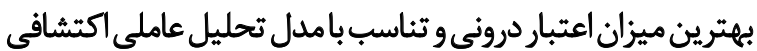

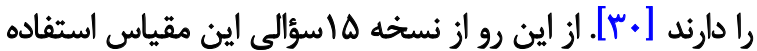

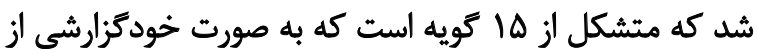

31. Implementation

32. Gollwitzer

33. The Short Defeat and Entrapment Scale

34. Interpersonal Needs Questionnaire

35. Hill

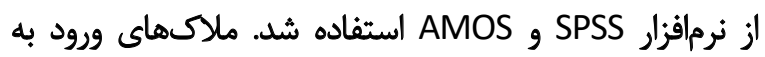

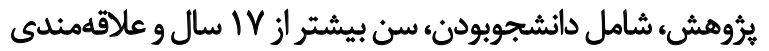

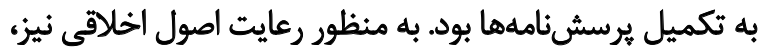

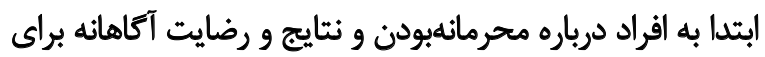

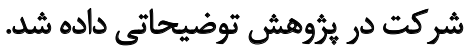

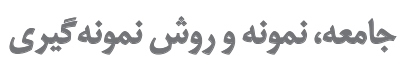

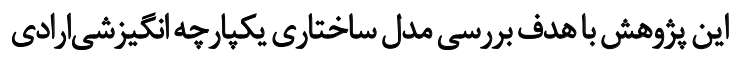

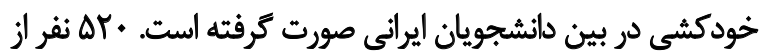

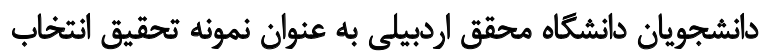

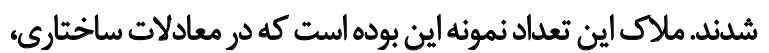

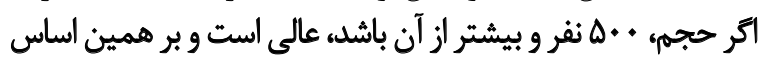
.

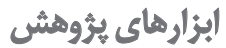

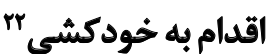

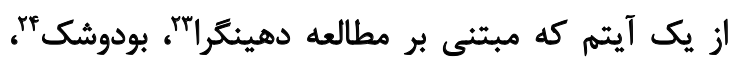

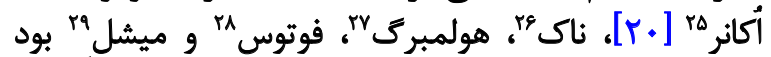

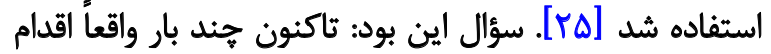

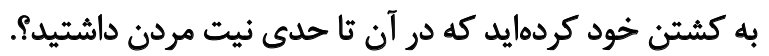

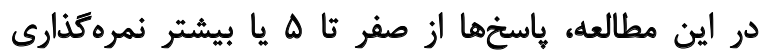

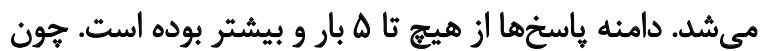

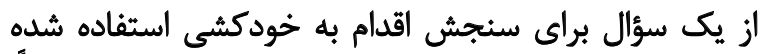

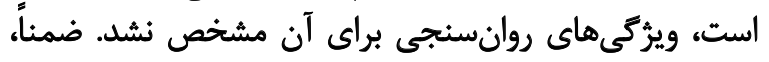

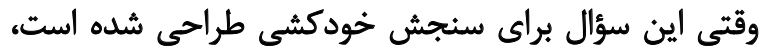
خودكشى را مي سنجند و روايى دارد

\section{"يرسش نامه رفتارهاى خودكشى -تجديدنظرشده"r}

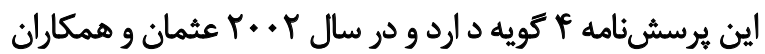

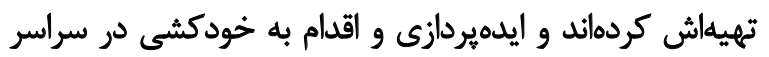

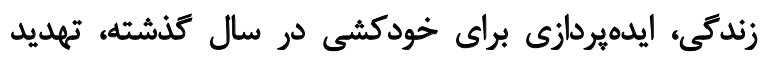

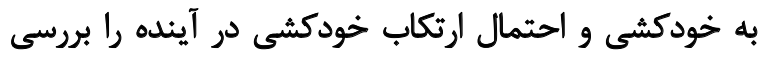

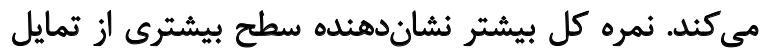

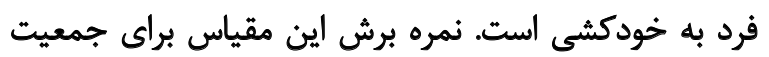

22. Suicide Attempt

23. Dhingra

24. Boduszek

25. O'Connor

26. Nock

27. Holmberg

28. Photos

29. Michel

30. The Suicide Behaviors Questionnaire-Revised (SBQ-R) 
ابعاد ساخته شده است، روايى دارد.

ظرفيت اكتسابشده خودادراكى براى خودكشى"

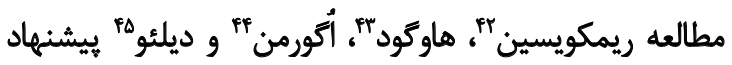

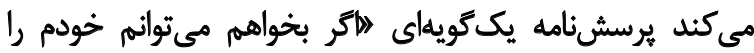

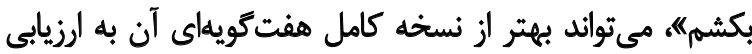

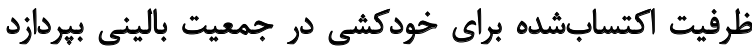

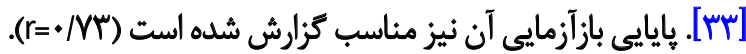

روش إجرا

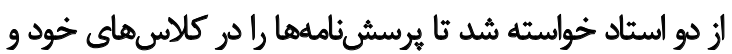

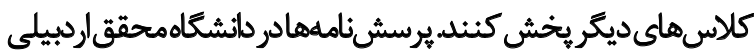

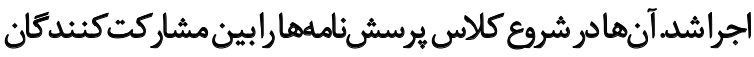

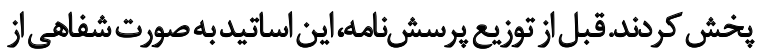

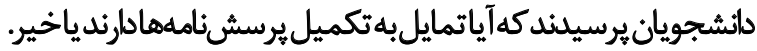

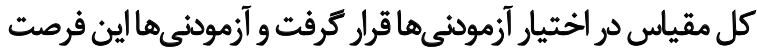

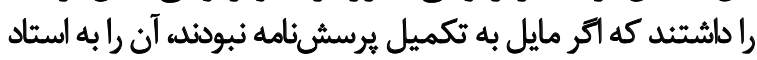

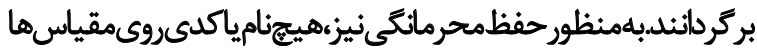

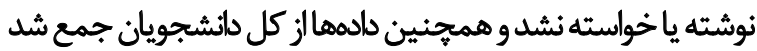

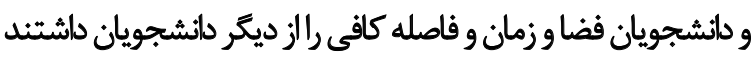

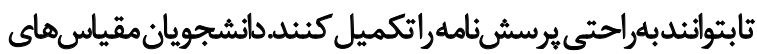

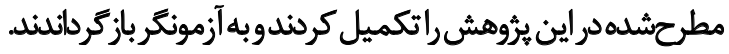

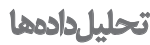

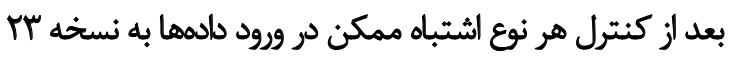

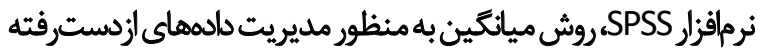

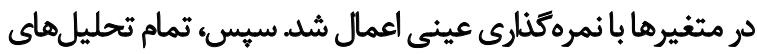

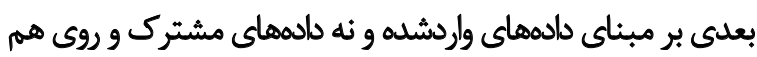

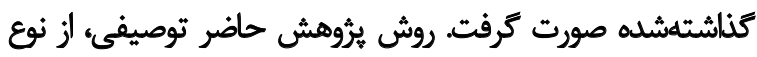

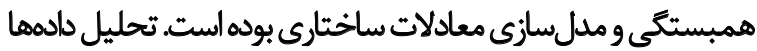

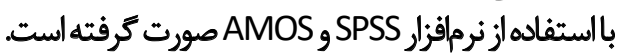

يافتهها

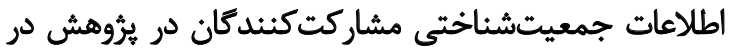

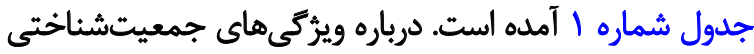

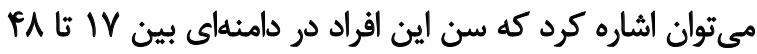

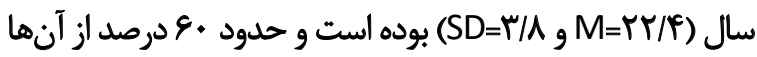

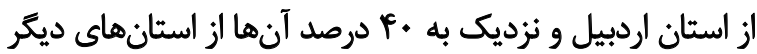

41. Self-Perceived Acquired Capability for Suicide

42. Rimkeviciene

43. Hawgood

44. O'Gorman

45. De Leo

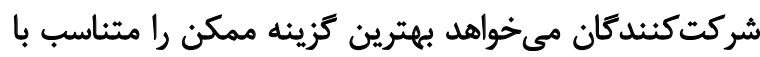

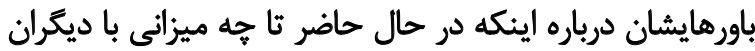

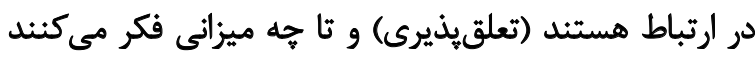

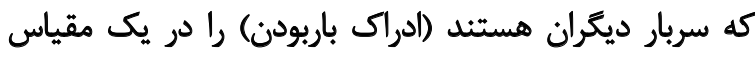

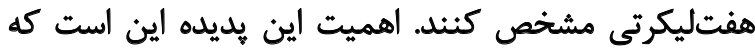

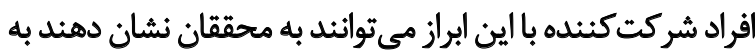

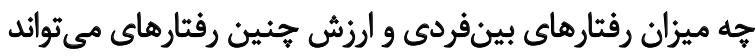

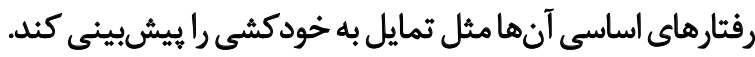

همجنين نمره بيشتردر اين مقياس به معناى ادراك سرباربودن

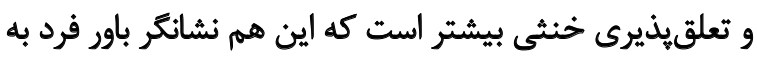

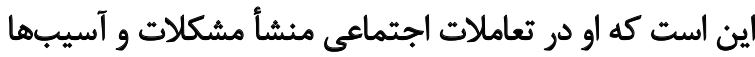

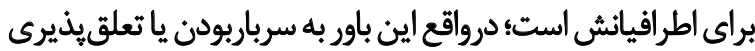

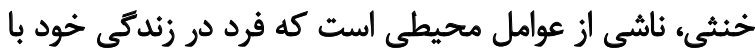

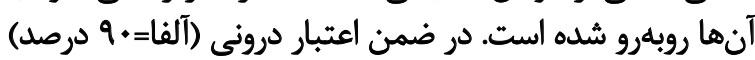

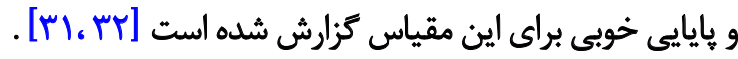

برسش بامه تكانشكرى"

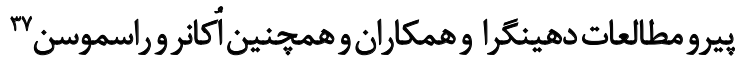

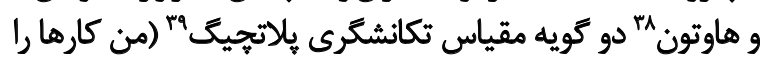

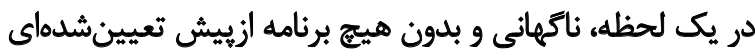

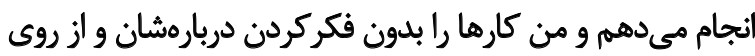

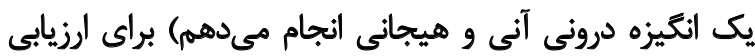

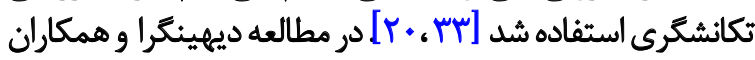

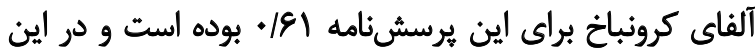

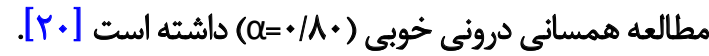

\section{يرسش نامه مواجهه با رفتار خودكشى"}

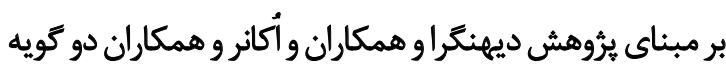

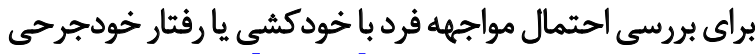

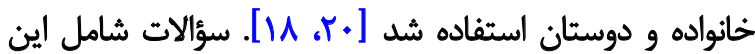

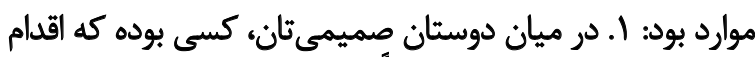

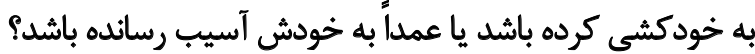

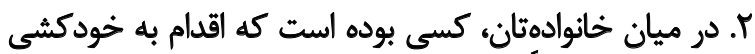

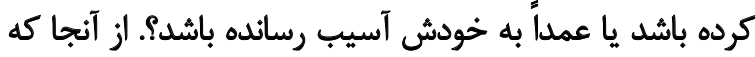

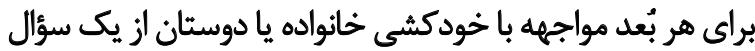

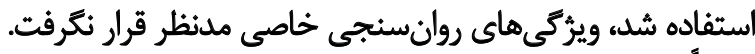

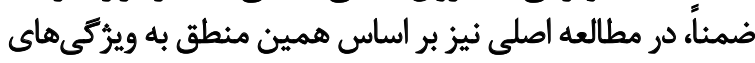

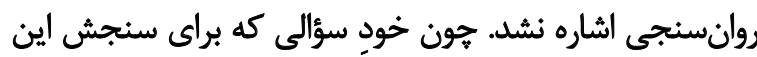

36. Impulsivity

37. Rasmussen

38. Hawton

39. Plutchik Impulsivity Scale

40. Exposure to suicidal behaviour 


$$
\text { تافكار و اقدام بيه خود كشي هستئد. }
$$

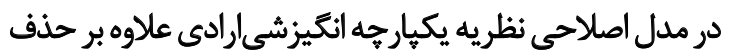

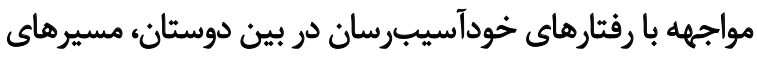

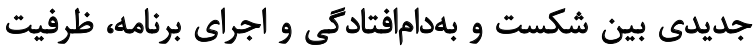

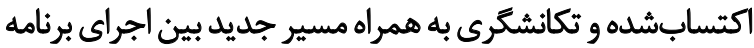

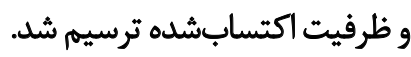

تصوير شماره ب مدل ساختّارى بيشنيهادى و ضرايب مسير در

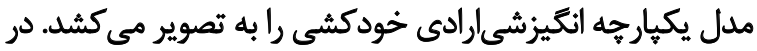

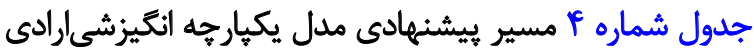

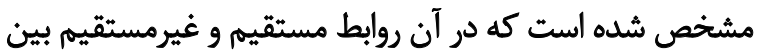

بودهاند. تنهها IV درصد از آنها كفتياند كه درآهد دارئد. بيشتر

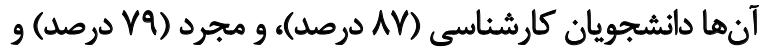

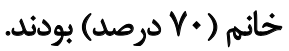

مدل ساختارى يُيشنهادى IMV در نمونهاي ايراني در

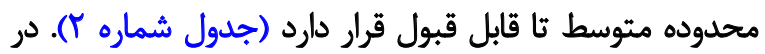

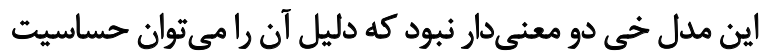

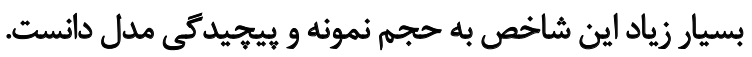
مدل اصلاحشده، شاخصهاي تناسب بسيار مناسبترى ارائه

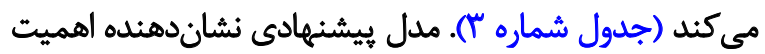

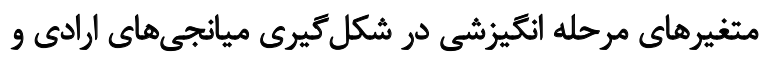

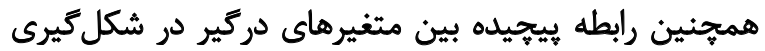

\begin{tabular}{|c|c|c|}
\hline فراوانى (درصد) & & \\
\hline $\begin{array}{l}r E P(V \cdot) \\
\operatorname{LEF}\left(r^{\prime} \cdot\right)\end{array}$ & زن & جنسيت \\
\hline $\begin{array}{l}\text { fil(vq) } \\
1.9(r)) \\
.\end{array}$ & مثاهل & وضعيت تأهل \\
\hline $\begin{array}{c}\operatorname{PAY}(A V) \\
E \cdot(11 / \Delta) \\
A(1 / \Delta)\end{array}$ & كارشناسى كارشئ & وضعيث تحصيلى \\
\hline $\begin{array}{l}\operatorname{Mrr}(A Y) \\
M(I V)\end{array}$ & شيكار & وضعيت درآمد \\
\hline $\begin{array}{l}\operatorname{PFI}(A \Delta) \\
\Delta \mathcal{N}(I I) \\
r I(Y)\end{array}$ & 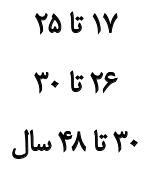 & وضعيت سنى \\
\hline $\begin{array}{l}r \mid r\left(\varepsilon_{0}\right) \\
r \cdot A\left(F_{0}\right)\end{array}$ & استان هاي ديكر اردييل & وضعيت بومىبودن \\
\hline
\end{tabular}

جدول ا. ويرّكى هاى جمعيتشناختي شركت كنيدكان يروهش

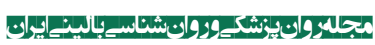

\begin{tabular}{|c|c|c|c|c|c|c|c|c|}
\hline CFA & NFI & RMSEA & AGFI & GFI & CMIN/df & df & $\mathrm{X}^{r}$ Test (P) & \\
\hline - /AFA & . ArA & .1111 & . IAOV & . $/ 9 \%$. & $|Y /| Q F$ & $M$ & $<+1 .+1$ & IMV \\
\hline
\end{tabular}

جدول r. شاخصهاى تثاسب مدل براى مدل ساختارى بيشنهادى

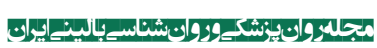

CFI GFI شاخص نيكويى برازش؛ CMIN/df : نسبت كاى اسكوئر به درجه آزادى؛ df: درجه آزادى؛ P: سطح معنادارى. 


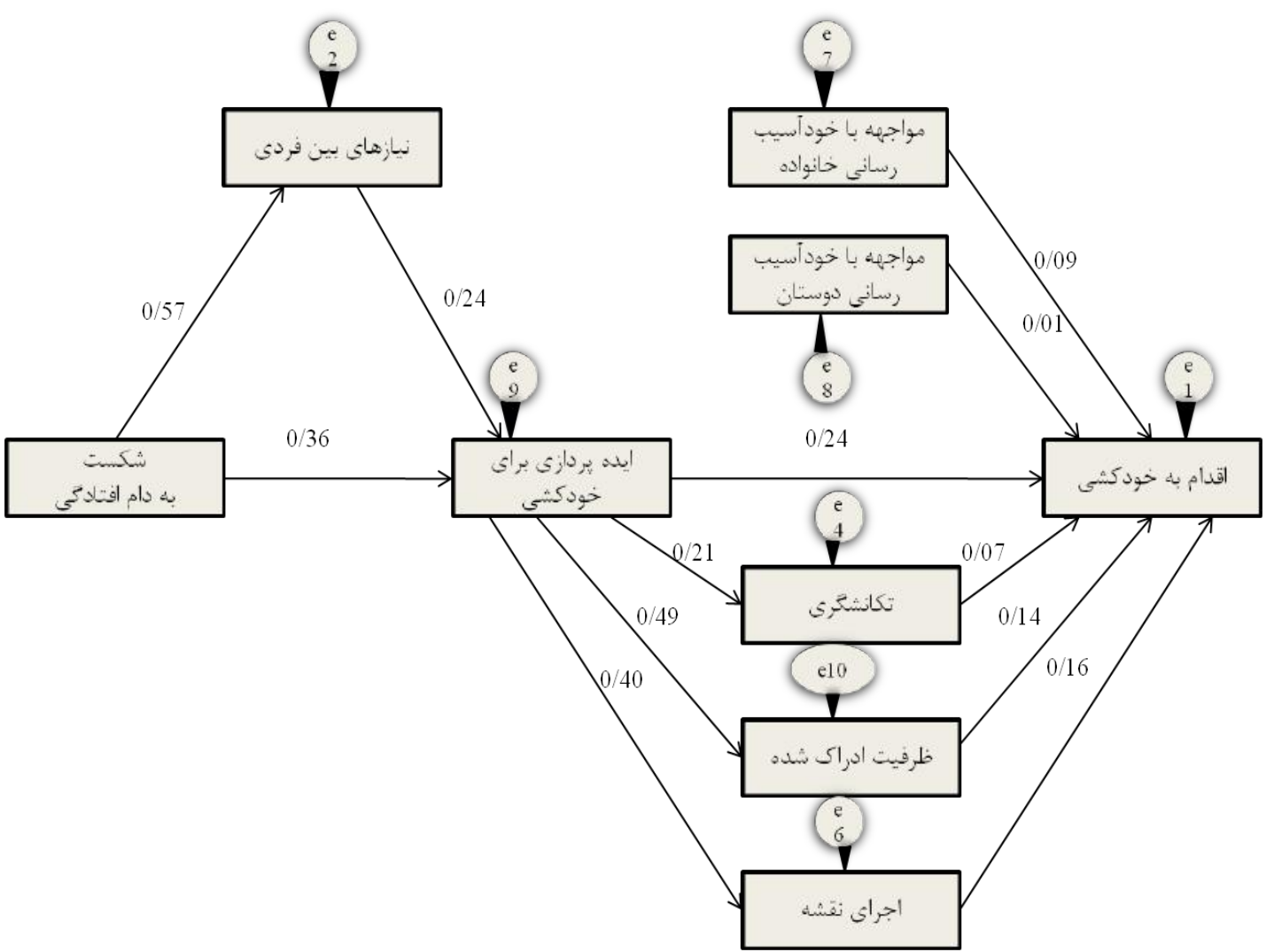

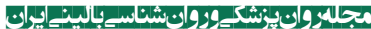

هدف از اين بروهش، بررسى ساختارى مدل يكيارجه

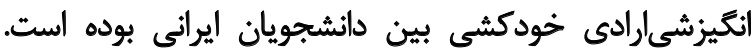

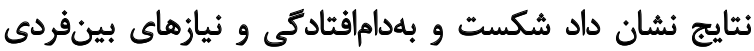

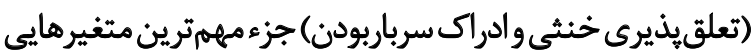

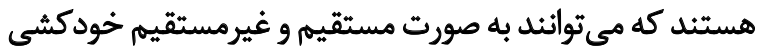

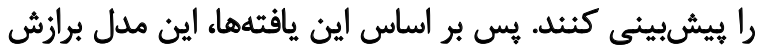

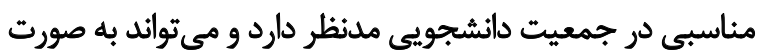

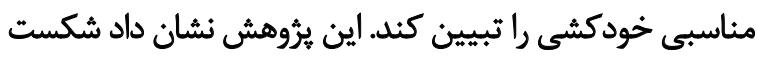

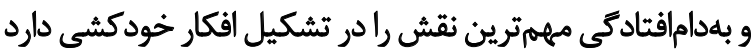

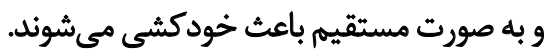

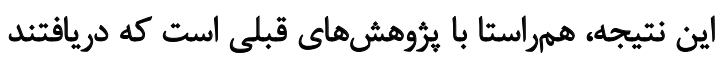

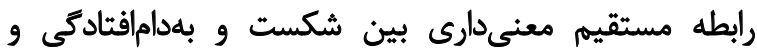

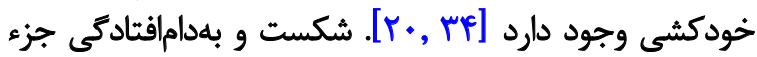

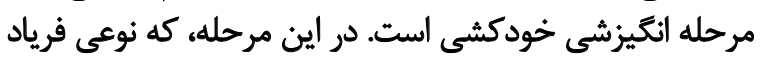

تصوير r. مدل ساختارى بيشنهادى و ضرايب مسير در مدل يكيارجه انكيزشىارادى

متغيرها نشان داده شده است. مسير بيشنهادى مدل يكيارجه

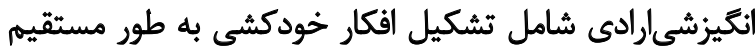

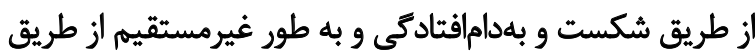

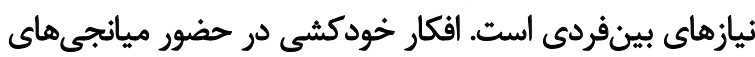

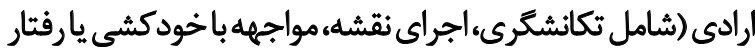

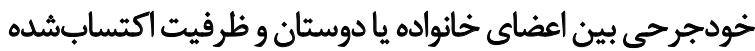
براى خودكشى) تبديل به اقدام به خودكشى مئشئودان

مطابق با اين مدل، شكست و بهدامافتادكى مهمترين نقش ردارئ

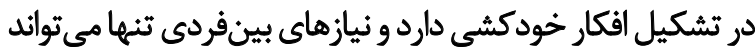

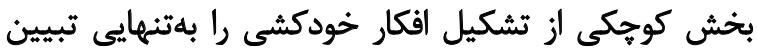

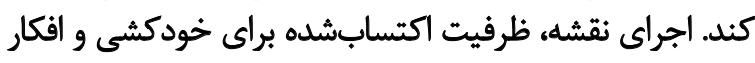

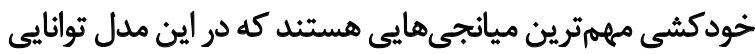

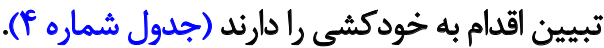

جدول r. شاخصهاى ثناسب مدل براى مدل ساختارى اصلاحشده

\begin{tabular}{ccccccccc}
\hline CFA & NFI & RMSEA & AGFI & GFI & CMIN/df & df & $X^{r}$ Test (P) & \\
\hline.$/ 9 \Delta \Delta$ &.$/ 9 \% \Lambda$ &.$/ \Lambda_{*}$ &.$/ 9 \% Y$ &.$/ 9 W$ & $9 / \Lambda \cdot$ & Ir & $<. / . .1$ & IMV \\
\hline
\end{tabular}


جدول F. ضريب تأثير مسيرهاى مستقيم، غيرمستقيم و كل در مدل يكيارجه انكيزشىارادى خودكثى

\begin{tabular}{|c|c|c|c|c|c|c|}
\hline Sig. & اثر كل & اثر مستقيم & اثر غيرمستقيم & متغير ييشبينىشونده & غيرمسيرهاي & | مسيرها \\
\hline $.1 . .1$ & &.$/ \Delta V$. & & نيازهاي بين فردى & & شكست و بهدام|فتًادكى \\
\hline $.1 \% 1$ & $\cdot / \Delta \cdot Y$ & . Wes & $. / 1, q 9$ & افكار خودكشى & نيازهاى بينفردى & شكست و بهدام|فقتادكى \\
\hline $.1+1$ & & 每 & & افكار خودكشى & & نيازهاى بينفردى \\
\hline $.1 .+1$ & & . MST & & مواجهه با رفتارهاى خودآسيبر رسان & & افكار خودكشى \\
\hline $.1 . .1$ & & .11 .8 & & مواجهه با رفتارهاى خودآسيبرسان & & افكار خودكشى \\
\hline.. .1 & &.$/ r \cdot 8$ & & تكانشكرى & & افكار خودكشى \\
\hline $.1+1$ & &.$/ p q$. & & ظرفيت اكتسابشده & & افكار خودكشى \\
\hline $.1 . .1$ & &.$/ \varphi \cdot \Delta$ & & اجراى نقشه & & افكار خودكشى \\
\hline $1 . .1$ &.$/ 299$ & A &.$/ 191$ & اقدام به خودكشى & & افكار خودكشى \\
\hline $.1++4$ & & $\% N$ & & اقدام به خودكشى & & مواجهه با رفتارهاى خودآسيبرسان در \\
\hline .MTT & & .1 .1 & & اقدام به خودكشى & & 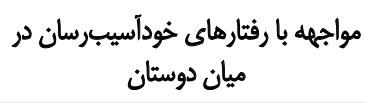 \\
\hline $.1 .5 q$ & & .1 .98 & & اقدام به خودكشى & & تكانشكرى \\
\hline .1 .1 & & אזו/. & & اقدام به خودكشى & & ظرفيت اكتسابشهد \\
\hline $.1 . .1$ & &.$/ 1 \Delta 9$ & & اقدام به خودكشى & & اجراى نقشه \\
\hline
\end{tabular}

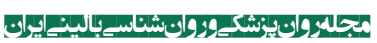

جون دارد درد را تجربه مى كند و راه فرارى براى درد نمىيابد

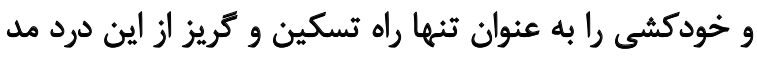

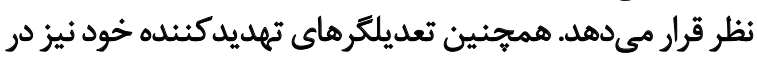

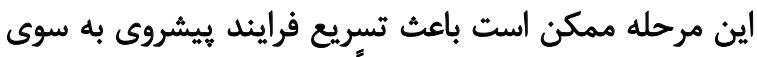

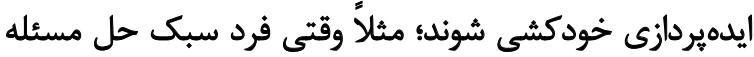

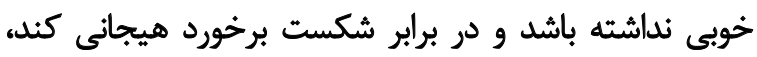

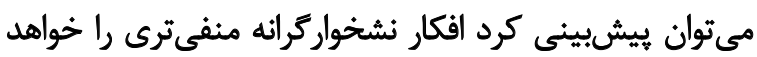

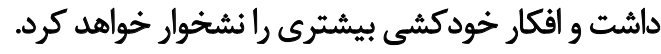

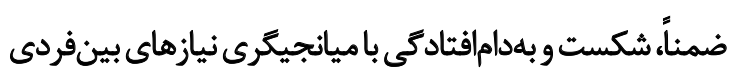

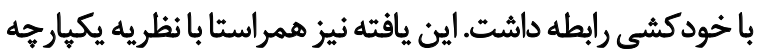

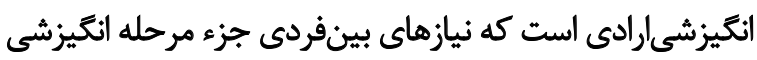

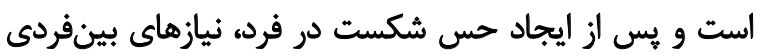

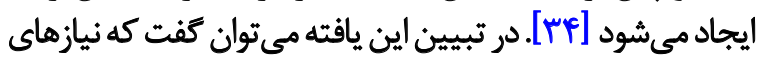

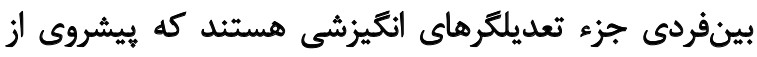

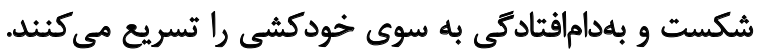

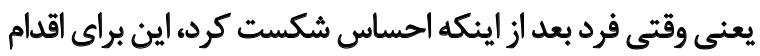

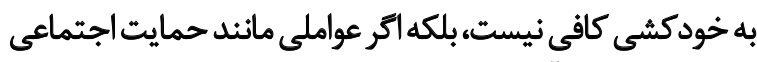

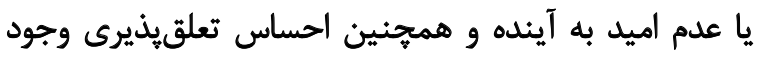

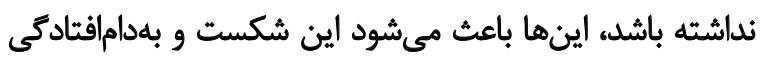

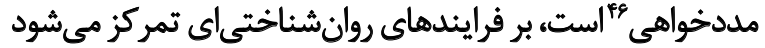

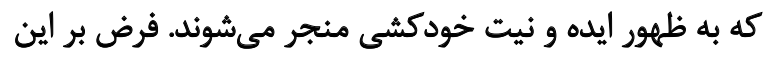

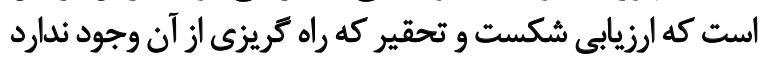

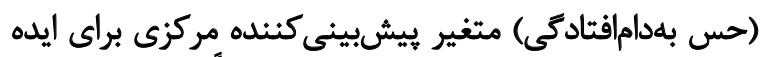

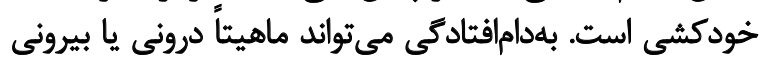

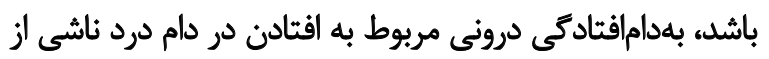

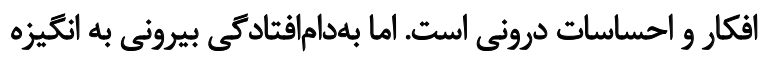

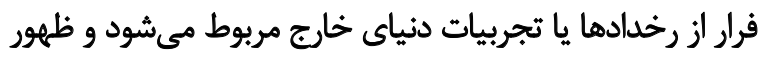

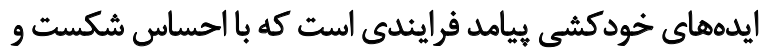

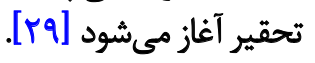
شكست و تحقير ممكن است با فقدان و طرد إجتماعي

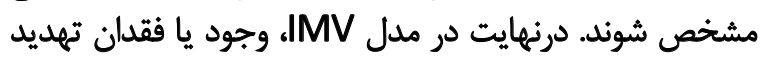

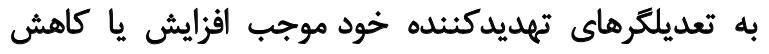

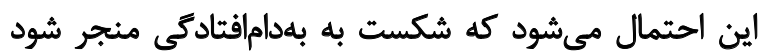

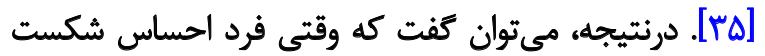

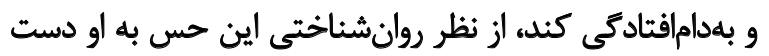

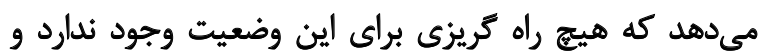
اينجاست كه ممكن است خودكشى را به عنوان كزينه بركزين وخيند،

46. Cry of help 
يعنى نيازهاى بينفردى (ادراى سرباربودن و تعلقيذيرى

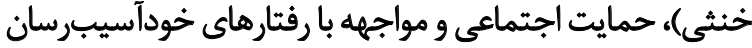

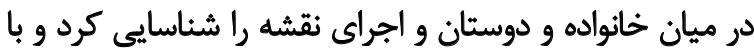

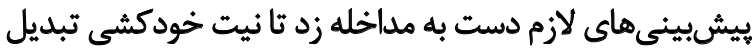
به اقدام به خودكشى نشود.

اين بثوهش نيز محدوديتهايي داشته است. بيشتر اين ابزارها

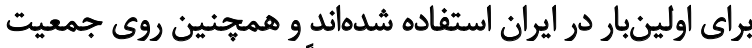

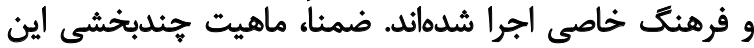

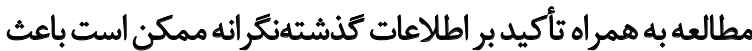

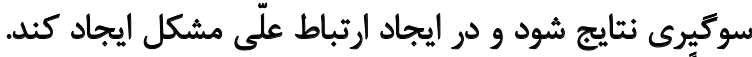

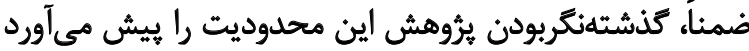

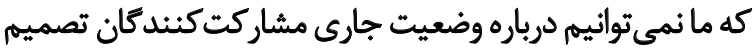

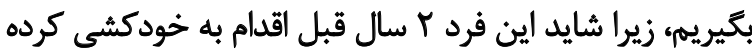

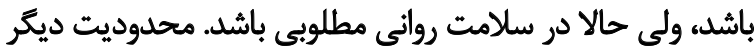

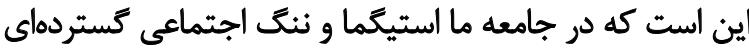

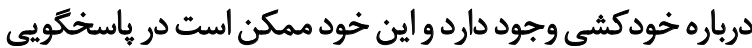
صادقانه مشاركت كنندكان و برآورد دقيق، مشكل آئ ايجاد كند.

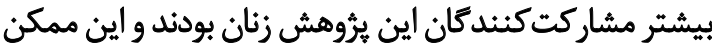

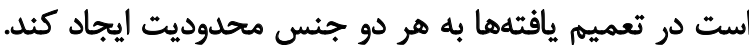

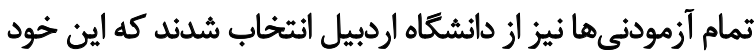

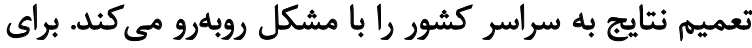

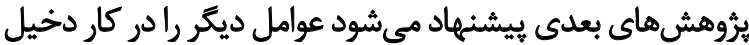

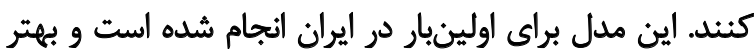

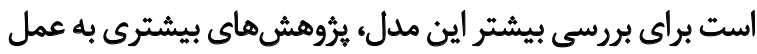

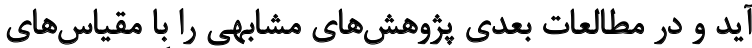

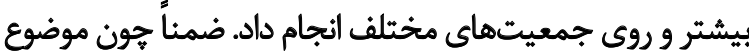

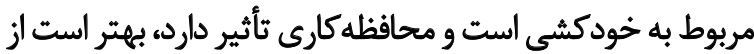
ابزارهاى ديكرى در كنار يرسش نامه استفاده كرد. درنهايت، بر اساس كاربرد مدل يكيارجه انتيزشىارادى

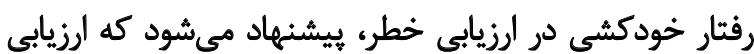

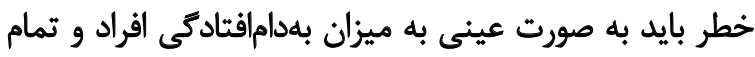

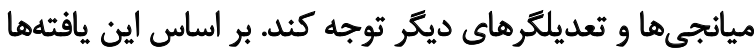

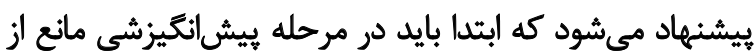

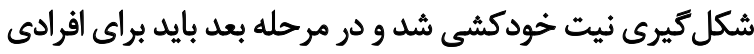

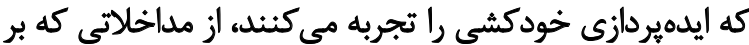

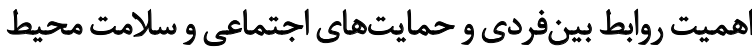
اطراف تأكيد دارند، استفاده كرد تا با اين حمايتهايت اجماعيت بتوان از اقدام به خودكشى يُشكيرى كرد.

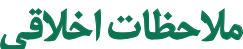

\section{بيروى أز اصول اخلاق يثوهش}

ورود به اين ثيروهش به صورت آكاهانه بوده است و آزمودنىها

$$
\text { تبديل به خودكشى شود. }
$$

يافتههاي اين ثئوهش نشان داد افكار خودكشى در حضور

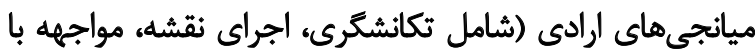

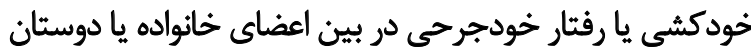

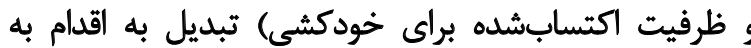

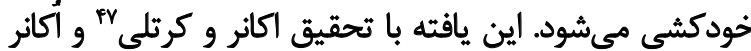

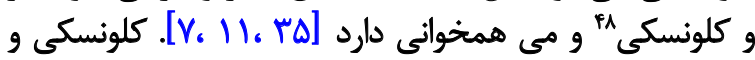

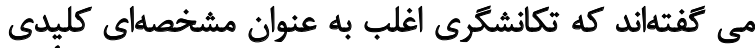

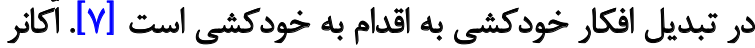

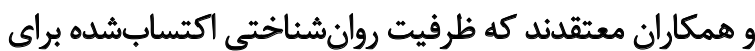

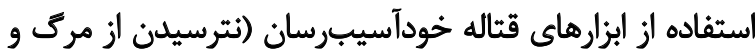

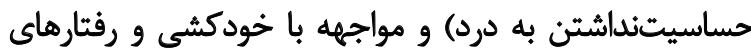

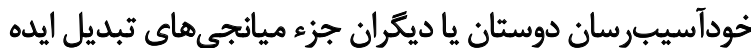

به رفتار خودكشى است [عبان].

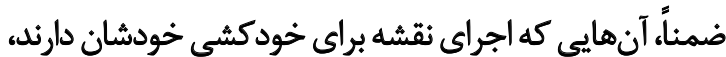

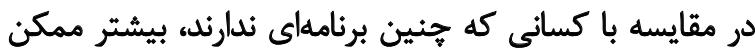

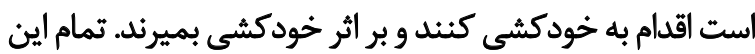

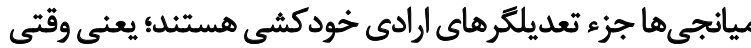

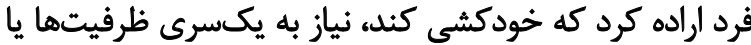

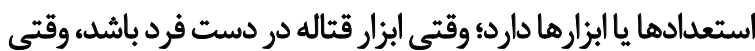

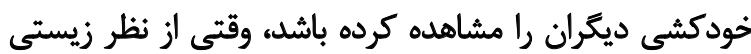

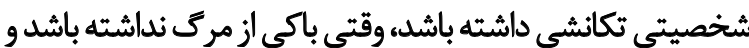

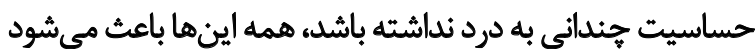
كه اين اراده تبديل به اقدام به خودكشى شود

\section{تَّيجه}

بر اساس يافتههاى اين تحقيق مى توان كفت كه اين تحقيق

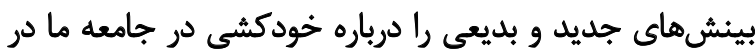

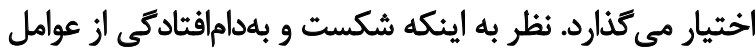

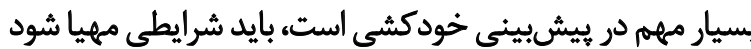

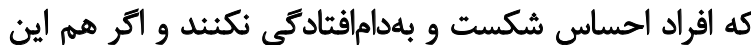

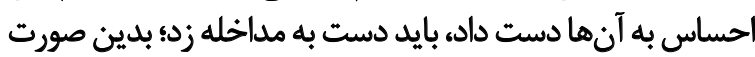

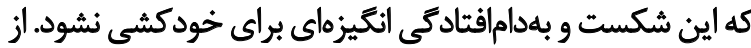

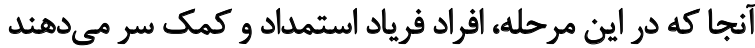

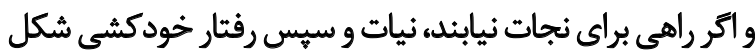

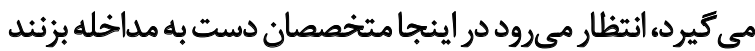

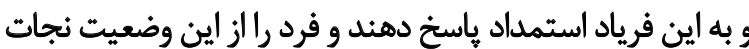

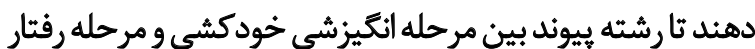
خود مشى قطع شود. ضمناً، در مرحله انكيزشى، مى توان رودى ميانجىها كار كرد؛

48. Klonsky 


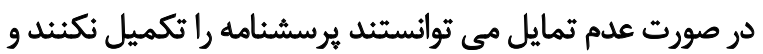

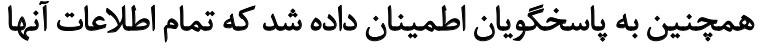

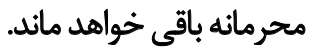

$$
\text { مامى مالى }
$$

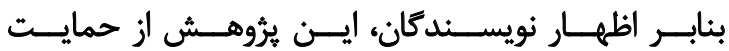

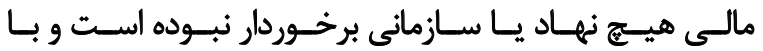

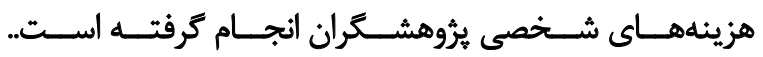

$$
\text { مشار كت نويسيندكَان }
$$

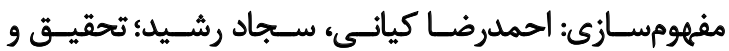

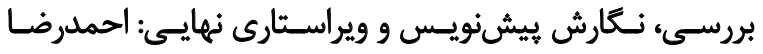

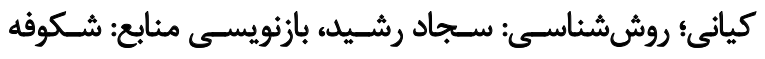

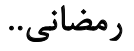

$$
\text { تعارض مثاقع }
$$

بنابر اظهار نويسندكان، اين مقاله تعارض منافع ندارد. 
[1] Fleischmann A, Bertolote JM, Wasserman D, De Leo D, Bolhari $\mathrm{J}$, Botega NJ, et al. Effectiveness of brief intervention and contact for suicide attempters: a randomized controlled trial in five countries. Bulletin of the World Health Organization. 2008; 86:703-9. [DOI:10.2471/BLT.07.046995] [PMID] [PMCID]

[2] Ma J, Batterham PJ, Calear AL, Han J. A systematic review of the predictions of the Interpersonal-Psychological Theory of Suicidal Behavior. Clinical Psychology Review. 2016; 46:34-45. [DOI:10.1016/j.cpr.2016.04.008] [PMID]

[3] Shirazi HR, Hosseini M, Zoladl M, Malekzadeh M, Momeninejad M, Noorian K, et al. Suicide in the Islamic Republic of Iran: An integrated analysis from 1981 to 2007. Eastern Mediterranean Health Journal. 2012; 18(6):607-13. [PMID]

[4] Hassanian-Moghaddam H, Zamani N. Suicide in Iran: The facts and the figures from nationwide reports. Iranian Journal of Psychiatry. 2017; 12(1):73-7. [PMID] [PMCID]

[5] Maltsberger JT. Reducing suicide: A national imperative. Journal of Nervous and Mental Disease. 2003; 191(9):623-4. [DOI:10.1097/01.nmd.0000087330.14485.41]

[6] Rashid S, Kiani, AR, Khorramdel K, Gholami F, Senobar L. The relationship between interpersonal psychological theory of suicide constructs (Loneliness, Perceived Social Support, Thwarted Belongingness and Burdensomeness) and suicidal behavior among Iranian students. Health Education \& Health Promotion. 2016; 4(2):35-48.

[7] Klonsky ED, May AM. The Three-Step Theory (3ST): A new theory of suicide rooted in the "ideation-to-action" framework. International Journal of Cognitive Therapy. 2015; 8(2):114-29. [DOI:10.1521/ijct.2015.8.2.114]

[8] Joiner T, Rudd MD. Suicide science: Expanding the boundaries. Berlin: Springer Science \& Business Media; 2007.

[9] Joiner, T. Why people die by suicide. Cambridge: Harvard University Press; 2007.

[10] Van Orden KA, Witte TK, Cukrowicz KC, Braithwaite SR, Selby EA, Joiner T. The interpersonal theory of suicide. Psychological Review. 2010; 117(2), 575-600. [DOI:10.1037/a0018697] [PMID] [PMCID]

[11] O'Connor R. Towards an integrated motivational-volitional model of suicidal behaviour. In: O'Connor, R., Platt, S. and Gordon, J. International Handbook of Suicide Prevention: Research, Policy and Practice. Glasgow: Wiley Blackwell; 2011. [DOI:10.1002/9781119998556.ch11]

[12] O'connor RC, Armitage CJ, Gray L. The role of clinical and social cognitive variables in parasuicide. British Journal of Clinical Psychology. 2006; 45(4):465-81. [DOI:10.1348/014466505X82315] [PMID]

[13] Ajzen I. The theory of planned behavior. Organizational behavior and Human Decision Processes. 1991; 50(2):179-211. [DOI:10.1016/0749-5978(91)90020-T]

[14] Schotte DE, Clum GA. Problem-solving skills in suicidal psychiatric patients. Journal of Consulting and Clinical Psychology. 1987; 55(1):49-54. [DOI:10.1037//0022-006X.55.1.49] [PMID]

[15] Williams M. Suicide and attempted suicide: Understanding the cry of pain. London: Penguin Books; 2002.

[16] Williams JMG, Crane C, Barnhofer T, Duggan D. Psychology and suicidal behaviour: Elaborating the entrapment model. Oxford: Oxford University Press 2013. [DOI:10.1093/ med/9780198529767.003.0005]

[17] O'Connor RC, Whyte MC, Fraser L, Masterton G, Miles J MacHale S. Predicting short-term outcome in well-being following suicidal behaviour: The conjoint effects of social perfectionism and positive future thinking. Behaviour Research and Therapy. 2007; 45(7):1543-55. [DOI:10.1016/j.brat.2006.11.006] [PMID]

[18] O'connor RC, Rasmussen S, Hawton K. Distinguishing adolescents who think about self-harm from those who engage in self-harm. The British Journal of Psychiatry. 2012; 200(4):330-5. [DOI:10.1192/bjp.bp.111.097808] [PMID]

[19] Van Orden KA, Witte TK, Gordon KH, Bender TW, Joiner Jr TE. Suicidal desire and the capability for suicide: Tests of the interpersonal-psychological theory of suicidal behavior among adults. Journal of Consulting and Clinical Psychology. 2008 76(1):72-83. [DOI:10.1037/0022-006X.76.1.72] [PMID] [PMCID]

[20] Dhingra K, Boduszek D, O'Connor RC. A structural test of the Integrated Motivational-Volitional model of suicidal behaviour. Psychiatry Research. 2016; 239:169-78. [DOI:10.1016/j.psychres.2016.03.023] [PMID]

[21] Ribeiro JD, Joiner TE. The interpersonal-psychological theory of suicidal behavior: Current status and future directions. Journal of Clinical Psychology. 2009; 65(12):1291-9. [DOI:10.1002/ jclp.20621] [PMID]

[22] Joiner Jr TE, Van Orden KA, Witte TK, Selby EA, Ribeiro JD, Lewis R, et al. Main predictions of the interpersonal-psychological theory of suicidal behavior: Empirical tests in two samples of young adults. Journal of Abnormal Psychology. 2009; 118(3):63446. [DOI:10.1037/a0016500] [PMID] [PMCID]

[23] Twenge JM, Catanese KR, Baumeister RF. Social exclusion and the deconstructed state: Time perception, meaninglessness, lethargy, lack of emotion, and self-awareness. Journal of Personality and Social Psychology. 2003; 85(3):409-23. [DOI:10.1037/00223514.85.3.409] [PMID]

[24] De Catanzaro D, Reproductive status, family interactions, and suicidal ideation: Surveys of the general public and highrisk groups. Ethology and Sociobiology. 1995; 16(5):385-94 [DOI:10.1016/0162-3095(95)00055-0]

[25] Nock MK, Holmberg EB, Photos VI, Michel BD. Self-injurious thoughts and behaviors interview: Development, reliability, and validity in an adolescent sample. Psychological Assessment. 2007; 19(3):309-17. [DOI:10.1037/t33713-000]

[26] Heidarali H. [Structural equation modeling using Laserl Software (Persian)]. Tehran: SAMT; 2016.

[27] Osman A, Bagge CL, Gutierrez PM, Konick LC, Kopper BA Barrios FX. The Suicidal Behaviors Questionnaire-Revised (SBQR): Validation with clinical and nonclinical samples. Assessment 2001; 8(4):443-54. [DOI:10.1177/107319110100800409] [PMID]

[28] Gollwitzer PM. Implementation intentions: Strong effects of simple plans. American Psychologist. 1999; 54(7):493-503. [DOI:10.1037/0003-066X.54.7.493]

[29] Gilbert P, Allan S. The role of defeat and entrapment (arrested flight) in depression: An exploration of an evolutionary view. Psychological Medicine. 1998; 28(3):585-98. [DOI:10.1017/ S0033291798006710] [PMID]

[30] Hill RM, Pettit JW. Perceived burdensomeness and suicide-related behaviors in clinical samples: Current evidence and future 
directions. Journal of Clinical Psychology. 2014; 70(7):631-43. [DOI:10.1002/jclp.22071] [PMID]

[31] Hawkins KA, Hames JL, Ribeiro JD, Silva C, Joiner TE, Cougle JR. An examination of the relationship between anger and suicide risk through the lens of the interpersonal theory of suicide. Journal of Psychiatric Research. 2014; 50:59-65. [DOI:10.1016/j. jpsychires.2013.12.005] [PMID]

[32] Van Orden KA, Cukrowicz KC, Witte TK, Joiner Jr TE. Thwarted belongingness and perceived burdensomeness: Construct validity and psychometric properties of the Interpersonal Needs Questionnaire. Psychological Assessment. 2012; 24(1):197-215. [DOI:10.1037/a0025358] [PMID] [PMCID]

[33] Rimkeviciene J, Hawgood J, O'Gorman J, De Leo D. Assessment of acquired capability for suicide in clinical practice. Psychology, Health \& Medicine. 2016; 21(8):954-63. [DOI:10.1080/135 48506.2015.1115108] [PMID]

[34] Dhingra K, Klonsky ED, Tapola V. An empirical test of the Three-Step Theory of suicide in UK University students. Suicide and Life-Threatening Behavior. 2019; 49(2):478-87. [DOI:10.1111/ sltb.12437] [PMID]

[35] O'Connor RC, Kirtley OJ. The integrated motivational-volitional model of suicidal behaviour. Philosophical Transactions of the Royal Society B. 2018; 373(1754):20170268. [DOI:10.1098/ rstb.2017.0268] [PMID] [PMCID]

[36] O'Connor RC, Ferguson E, Scott F, Smyth R, McDaid D, Park $\mathrm{AL}$, et al. A brief psychological intervention to reduce repetition of self-harm in patients admitted to hospital following a suicide attempt: A randomized controlled trial. The Lancet Psychiatry. 2017; 4(6):451-60. [DOI:10.1016/S2215-0366(17)30129-3] 